\title{
Loss of COUP-TFI Alters the Balance between Caudal Ganglionic Eminence- and Medial Ganglionic Eminence-Derived Cortical Interneurons and Results in Resistance to Epilepsy
}

\author{
Simona Lodato, ${ }^{1,2,3 *}$ Giulio Srubek Tomassy, ${ }^{1,3 *}$ Elvira De Leonibus, ${ }^{1 *}$ Yoryani G. Uzcategui, ${ }^{4,5}$ Gennaro Andolfi, ${ }^{1}$ \\ Maria Armentano, ${ }^{1}$ Audrey Touzot, ${ }^{6,7}$ Jose M. Gaztelu, ${ }^{5}$ Paola Arlotta, ${ }^{3}$ Liset Menendez de la Prida, ${ }^{4}$ \\ and Michèle Studer ${ }^{1,2,6,7}$ \\ ${ }^{1}$ Telethon Institute of Genetics and Medicine, Developmental Disorders Program, and 'European School of Molecular Medicine, 80131 Naples, Italy, \\ ${ }^{3}$ Department of Stem Cell and Regenerative Biology and Harvard Stem Cell Institute, Harvard University, Cambridge, Massachusetts 02138, ${ }^{4}$ Instituto \\ Cajal-Consejo Superior de Investigaciones Científicas, 28002 Madrid, Spain, ${ }^{5}$ Hospital Ramón y Cajal, 28034 Madrid, Spain, and ${ }^{6}$ Inserm, Unit 636, and \\ 7University of Nice Sophia-Antipolis, F-06108 Nice, France
}

In rodents, cortical interneurons originate from the medial ganglionic eminence (MGE) and caudal ganglionic eminence (CGE) according to precise temporal schedules. The mechanisms controlling the specification of CGE-derived interneurons and their role in cortical circuitry are still unknown. Here, we show that COUP-TFI expression becomes restricted to the dorsal MGE and CGE at embryonic day 13.5 in the basal telencephalon. Conditional loss of function of COUP-TFI in subventricular precursors and postmitotic cells leads to a decrease of late-born, CGE-derived, VIP (vasoactive intestinal peptide)- and CR (calretinin)-expressing bipolar cortical neurons, compensated by the concurrent increase of early-born MGE-derived, PV (parvalbumin)-expressing interneurons. Strikingly, COUP-TFI mutants are more resistant to pharmacologically induced seizures, a phenotype that is dependent on GABAergic signaling. Together, our data indicate that COUP-TFI controls the delicate balance between MGE- and CGE-derived cortical interneurons by regulating intermediate progenitor divisions and ultimately affecting the activity of the cortical inhibitory circuitry.

\section{Introduction}

In rodents, interneurons are born in basal telencephalic structures, such as the medial ganglionic eminence (MGE), the caudal ganglionic eminence (CGE), and the preoptic area from which they migrate to populate the cortex (Corbin et al., 2001; Marín and Rubenstein, 2001, 2003). Various transplantation and fatemapping experiments have shown that such a wide diversity is intimately dependent on their birth date and location. Early-born interneurons originate from the MGE and produce mainly the

Received Dec. 17, 2010; accepted Jan. 17, 2011.

This work was supported by the Italian Telethon Foundation (M.S.), the Spanish Fundación Alicia Koplowitz (L.M.d.I.P.), the Harvard Stem Cell Institute (P.A.), and European Community FP6 STREP Contract 005139 (INTERDEVO) (M.S., L.M.d.I.P.). S.L. is the recipient of a predoctoral fellowship of the European School of Molecular Medicine (Naples, Italy). We are grateful to G. Fishell, 0. Marín, M. Ross, and M. Nieto for helpful comments on this manuscript. We thank F. Guillemot for cyclin D1 and cyclin D2 plasmids; V. Pachnis for the VIP plasmid and the Lhx6 antibody; J. L. R. Rubenstein for the Nkx2.1, Gad67, and somatostatin plasmids; R. Rusconi for designing the cell counting algorithm developed in Matlab; M. Mancuso, F. Russo, A. I. Merlino for technical assistance; and M. Giordano for animal husbandry. L.M.d.I.P. and M.S. jointly coordinated and designed this work.

*S.L., G.S.T., and E.D.L. contributed equally to this work.

Correspondence should be addressed to Michèle Studer at the above address. E-mail: michele.studer@unice.fr.

E. De Leonibus's present address: Consiglio Nazionale delle Ricerche Institute of Genetics and Biophysics “Adriano Buzzati Traverso," 80131 Naples, Italy.

M. Armentano's present address: Department of Veterinary Morphophysiology, University of Turin, 10095 Grugliasco, Turin, Italy.

DOI:10.1523/JNEUROSCI.6580-10.2011

Copyright $\odot 2011$ the authors $\quad 0270-6474 / 11 / 314650-13 \$ 15.00 / 0$ parvalbumin (PV)- and somatostatin (SST)-expressing subtypes, which contribute primarily to deep cortical layers; late-born interneurons derive predominantly from the CGE, generate calretinin (CR)- and vasoactive intestinal peptide (VIP)-expressing interneurons, and preferentially occupy superficial cortical layers (Nery et al., 2002; Xu et al., 2004; Butt et al., 2005, 2008; Cobos et al., 2006; Fogarty et al., 2007; Miyoshi et al., 2007, 2010).

Understanding the spatial and temporal mechanisms of GABAergic cortical interneuron determination is attracting much interest in the last few years. Transcription factors expressed either broadly within the two eminences or predominantly in one eminence play a fundamental role in the specification and maturation of these cells (Batista-Brito and Fishell, 2009). Whereas loss of Dlx1/Dlx2 function results in a massive decrease of neocortical GABAergic interneurons at birth (Anderson et al., 1997), mice lacking only the $D l x 1$ gene show reduction of CR- and SST-expressing interneurons without affecting the PV-expressing population (Cobos et al., 2005). These mice have cortical dysrhythmia and generalized seizures. Early removal of the transcription factor $N k \times 2.1$, restricted to the MGE domain, results in a molecular and cellular switch of MGEderived cortical interneurons (PV- and SST-positive subpopulations) to CGE-derived neurons (VIP- and CR-expressing cells), leading ultimately to seizure activities (Butt et al., 2008). Down- 
stream of Nkx2.1, the transcription factor Lhx6 is involved in the specification and migration of PV- and SST-expressing interneurons (Liodis et al., 2007), whereas Sox6, acting downstream to Lhx6 (Batista-Brito et al., 2009), is required for the correct balance of PV-, SST-, and neurotransmitter peptide Y (NPY)expressing subpopulations (Azim et al., 2009; Batista-Brito et al., 2009). Loss of function of Sox6 results in a dramatic reduction of PV- and SST-expressing interneurons, whereas NPY + interneurons outnumber the normal level without affecting the CGEderived CR- and VIP-expressing subpopulations. Interestingly, similarly to Dlx1 and Nkx2.1 mutant mice, Sox6 mutants suffer from generalized epileptic seizures (Batista-Brito et al., 2009).

Although many reports have characterized genetic determinants of MGE-derived cortical interneurons, little is known about the regional and cell type specification of CGE-derived interneurons, which comprise $\sim 30 \%$ of all cortical interneurons with an unexpected higher diversity than previously anticipated (Miyoshi et al., 2010). The orphan nuclear receptor COUP-TFII shows restricted expression in the CGE (Kanatani et al., 2008; Willi-Monnerat et al., 2008) and, together with COUP-TFI, is required for the caudal migration of cortical interneurons (Tripodi et al., 2004; Kanatani et al., 2008). Moreover, in Nkx2.1 conditional mutant mice, in which a high number of CR- and VIP-expressing cortical interneurons are generated, COUP-TFII is ectopically expressed in the MGE (Butt et al., 2008). Together, these reports strongly suggest that COUP-TF members might be directly involved in the migration and specification of CGEderived cortical interneurons.

Here, we show that COUP-TFI expression becomes gradually restricted to the caudal basal telencephalon. Conditional inactivation of COUP-TFI using a pan-interneuronal Cre mouse line leads to increased PV-expressing interneurons and decreased VIP- and CR-expressing bipolar neurons, without affecting the total number of cortical GABAergic cells. Interestingly, COUPTFI conditional embryos show increased cellular proliferation predominantly in the MGE as well as enhanced expression of the cell cycle gene cyclinD2, known to establish the proper density of PV-expressing interneurons (Glickstein et al., 2007b). Strikingly, we found no discernible electroencephalographic (EEG) abnormalities, but, instead, COUP-TFI conditional mice are more resistant to pharmacologically induced seizures, a GABA-dependent property. Together, our data indicate an intrinsic property of COUP-TFI in regulating the balance between MGE- and CGEderived cortical interneurons, thus contributing to the proper formation of the cortical local inhibitory circuitry.

\section{Materials and Methods}

Mice. COUP-TFf $f^{f l o x /+}$ mice were generated as previously reported (Armentano et al., 2007) and propagated by backcrossing to C57BL/6 inbred mice. Homozygous COUP-TFF flox/flox mice were obtained by intercrossing heterozygous COUP-TFf flox/+ mice and mated to the Dlx5/6-CreIRES-GFP transgenic line (Stenman et al., 2003), a kind gift from K. Campbell (Children's Hospital Research Foundation, Cincinnati, $\mathrm{OH}$ ), to generate conditional COUP-TFI CKO-Dlx5/6 embryos and mice (COUP-TFf flox/flox homozygous-Dlx5/6 Cre heterozygous). Throughout the study, we used as controls [referred also as wild type (WT)] either heterozygous (COUP-TFF flox/+ $)$, homozygous (COUP-TFf flox/flox $)$, or Dlx5/6-Cre-positive (Cre+ ) mice, since they show no phenotypic abnormalities, as previously described (Stenman et al., 2003; Armentano et al., 2007). We found no phenotypic differences between male and female mutant mice. Genotyping was performed as previously described (Stenman et al., 2003; Armentano et al., 2007). Midday of the day of the vaginal plug was embryonic day 0.5 (E0.5). All experiments were conducted following guidelines of the Institutional Animal Care and Use Committee, Cardarelli Hospital (Naples, Italy).

Immunocytochemistry and in situ hybridization. Mice were perfused with $4 \%$ buffered paraformaldehyde (PFA), and decapitated heads (E12.5-E14.5) or brains [postnatal day 21 (P21)] were postfixed in 4\% PFA for $12 \mathrm{~h}$ at $4^{\circ} \mathrm{C}$. Brains were either sectioned on a vibratome at $50 \mu \mathrm{m}$ or cryosectioned in OCT medium (Tissue-Tek) at $20 \mu \mathrm{m}$. Vibratome sections were processed free floating, and standard nonradioactive in situ hybridization and immunofluorescence protocols were used. In situ hybridization and combined immunohistochemistry were performed as previously described (Tripodi et al., 2004). The following primary antibodies were used: COUP-TFI (rabbit; 1:500) (Tripodi et al., 2004), bromodeoxyuridine (BrdU) (mouse; 1:300; Sigma-Aldrich), Ki67 (rat; 1:250; Dako), GABA (rabbit; 1:1000; Sigma-Aldrich), NPY (rabbit; 1:3000; Diasorin), calretinin (rabbit; 1:5000; Swant), parvalbumin (mouse; 1:1000; Millipore), Lhx6 (rabbit; 1:500; kind gift from V. Pachnis), and green fluorescent protein (GFP) (rabbit; 1:1000; Millipore). The following secondary antibodies were used: 1:400, Alexa Fluor $488 \alpha$-rabbit; Alexa Fluor $594 \alpha$-rabbit; Alexa Fluor $594 \alpha$-mouse (Invitrogen); 1:200, biotinylated goat anti-rabbit (Vector Laboratories). Nonradioactive in situ hybridization on 16 - to 20 - $\mu$ m-thick cryostat sections were performed as previously described (Armentano et al., 2007). Antisense RNA probes were labeled using a DIG-RNA labeling kit (Roche). The following probes were used: COUP-TFI, Gad67, SST, VIP, CyclinD2, and Nkx2.1.

BrdU birthdating. Timed-pregnant females received a single intraperitoneal injection of $\mathrm{BrdU}(50 \mathrm{mg} / \mathrm{kg}) 1 \mathrm{~h}$ before being killed, and embryos were collected at E13.5. WT and COUP-TFI CKO-Dlx5/6 embryos were examined for BrdU-positive cell distribution in the MGE and CGE, as previously described (Tomassy et al., 2010). Sections from rostral to intermediate levels (see Fig. $1 B, C$ ), where the MGE is still distinct, and from caudal to caudalmost levels where the CGE (see Fig. $1 D$ ) is clearly defined, were photographed at $10 \times$ magnification on a Leica DM5000B equipped with Leica IM image management software (Leica Microsystems), and then imported into Adobe Photoshop for counting. The percentage of BrdU-positive cells was calculated as the ratio of double BrdU/ 4',6'-diamidino-2-phenylindole (DAPI)-positive cells divided by the total number of DAPI-positive cells.

Electroencephalographic recordings. Freely moving mice aged between 7 and 13 weeks were tested for spontaneous EEG activity. Animals were anesthetized with ketamine $(100 \mathrm{mg} / \mathrm{kg})$ and xylazine $(10 \mathrm{mg} / \mathrm{kg}$, i.p.) and placed in a stereotaxic apparatus. Cortical EEG recordings were obtained from $100-\mu \mathrm{m}$-wide tungsten wires implanted over the parietal cortex at $0.2-0.4 \mathrm{~mm}$ depth from the cortical surface using bone cement. Two screws were used as a reference and ground at the occipital region. In a group of animals, two separate wires were bilaterally implanted in the dorsal hippocampus to obtain simultaneous cortical and hippocampal EEG activity. After recovering from surgery, EEG was recorded from individual mice over several days, for a maximum of $2 \mathrm{~h}$ per day, by using a Grass EEG Neurodata system. Total recording time per mouse ranged from 18 to 36 h over the course of 12-20 d. Animals were simultaneously videotaped for identifying different behavioral states. EEG recordings during awake states were bandpass FIR filtered between 1 and $200 \mathrm{~Hz}$ and sampled at $500 \mathrm{~Hz}$. After completing EEG recordings, brains were fixed in $4 \%$ PFA for electrode placement verification.

EEG data were analyzed with Spike 2 and routines developed in Matlab. The spectral power was calculated using the fast Fourier transform with Hamming window and 1024 points for artifact-free recording windows of 10 min duration. To study the frequency content independently of amplitude changes, each EEG spectrum was normalized by the total power in the $1-100 \mathrm{~Hz}$ band. The mean power spectrum for each group is represented together with the $95 \%$ confidence intervals. To look at spectral differences between types of oscillations, four different frequency bands were defined: $1-4 \mathrm{~Hz}$ (delta), $4-10 \mathrm{~Hz}$ (theta), $10-40 \mathrm{~Hz}$ (beta), and 40-100 Hz (gamma). The power spectrum value for each band was calculated by integrating the normalized power spectrum in the corresponding frequency interval. For statistical comparisons, we first tested data for normal distribution using the Kolmogorov-Smirnov test. Power spectral values in a logarithmic scale were normally distributed 
except for the $40-100 \mathrm{~Hz}$ gamma band. Thus, to quantify statistical differences, spectra from the two experimental groups were compared using a nonparametric test for multiple comparisons (Mann-Whitney $U$ test). To compare the spectral distribution at the delta, theta, and beta bands, a two-way ANOVA was used with the factors group and gender. Differences in the gamma band were compared using the Kruskal-Wallis nonparametric test for the group and for the gender independently.

Pharmacological seizure induction. For pilocarpine-induced seizures, mice were intraperitoneally injected with lithium chloride $(423 \mathrm{mg} / \mathrm{kg})$ between 18 and $20 \mathrm{~h}$ before $100 \mathrm{mg} / \mathrm{kg}$ pilocarpine hydrochloride. Pentylenetetrazole (PTZ) (Sigma-Aldrich) was administered subcutaneously at a dose of $90 \mathrm{mg} / \mathrm{kg}$. We chose a suprathreshold dose of PTZ to induce clonic seizures independently on the gender and with uniform latency within groups (Löscher et al., 1991). In a group of PTZ experiments, we pretreated mice with the $\mathrm{GABA}_{\mathrm{B}}$ receptor antagonist (3aminopropyl)(diethoxymethyl)phosphinic acid hydrate (GCP 35348) (Sigma-Aldrich) at a dose of $136 \mathrm{mg} / \mathrm{kg}$. Mice injected with PTZ and not pretreated with GCP 35348 were divided in two groups, one-half not injected and one-half injected with vehicle. Animals were observed for 45 min after the PTZ injection. Three different stages of seizure activity were scored: stage 1 , body and tail rigidity; stage 2 , characterized by whole-body clonus; and stage 3 , characterized by generalized tonic-clonic hindlimb extensions.

Cell counting. For the quantification of interneuron subpopulations in COUP-TFI CKO-Dlx5/6 and wild-type cortices, three coronal anatomically matched sections within the sensorimotor area in the rostrocaudal axis (bregma, from 0.50 to $-0.34 \mathrm{~mm}$ ) were selected from littermate mice and processed by immunocytochemistry to detect GABA, PV, NPY, and CR, and in situ hybridization to detect SST and VIP transcript $(n=$ 3 COUP-TFI CKO-Dlx5/6; $n=3$ wild type, 6 hemispheres per area, for each mouse at P21). Digital boxes of fixed width were superimposed on each coronal section and they were divided into 10 sampling areas (bins) with a dorsoventral extent from the pial surface to the white matter (corpus callosum). Deep layer were assigned to bin 1-5 and superficial layers were assigned to bin 6-10, based on anatomical features. Cell detection and counting were performed using a customized imaging processing software. Bipolar cells were identified manually. The automated algorithm has been developed in Matlab (version 7.6; The MathWorks) by an investigator blinded to the study design and consisted in a series of processing steps. In the first one, original fluorescent or brightfield images, taken at $4 \times$ magnification, are corrected for uneven illumination and background noise. Image segmentation is then obtained through edge detection, in which minimum and maximum levels of the intensity gradient are established a priori for each staining analyzed and kept constant throughout the counting analysis. Then, morphological characteristics were added to the filtering process. The eccentricity parameter-where 1 indicates a linear shape and 0 indicates a circular shape-was used to identify cell bodies, by using a threshold values established a priori. In combination with the eccentricity parameter, thresholds for the cell size were established a priori based on the normal distribution of cell area for each staining analyzed. In addition, the area parameter was used to discriminate single cells within clusters. For the quantification of the percentage of interneuron subtypes that express COUP-TFI in P8 and P21 wild-type cortices, three coronal sections spanning within the somatosensory area in the rostrocaudal axis (bregma, $-0.34 \mathrm{~mm}$ ) were selected from littermate mice $(n=3,6$ hemispheres per area, for each wild-type mouse at P8 and P21). Digital boxes of fixed width were superimposed on each coronal section and cell counting was performed on images taken at $10 \times$ magnification by an investigator blinded to the study design. Counting criteria were established a priori.

Data analysis. All cell counting data and the graphs were constructed using Microsoft Excel software. For each animal, a mean value was calculated from all the sections counted, and for each genotype, a mean value was obtained by pooling the means of the three sampled animals. EEG data were analyzed using the Mann-Whitney $U$ test for comparison of the power spectra between groups and an ANOVA or a Kruskal-Wallis test for comparison of the four different frequency bands between group and gender (see above, Electroencephalographic recordings). All behavioral pharmacological data were analyzed using either one-way ANOVA (genotype, two levels: WT and CKO) or two-way ANOVA [genotype (two levels: WT and CKO) and pretreatment (two levels: vehicle and CGP 35348)]. Post hoc analysis was performed using Duncan's post hoc test. All error bars represent the SEM. Statistical significance was determined using two-tailed Student's $t$ tests $\left({ }^{\star} p<0.05\right.$; $\left.{ }^{* *} p<0.01\right)$.

\section{Results \\ COUP-TFI is preferentially expressed in the dorsal MGE, in the CGE, and in a subpopulation of mature cortical interneurons}

To investigate the function of COUP-TFI in the development of cortical GABAergic interneurons, we first characterized its expression profile in the developing ganglionic eminences. From E10.5 to E12.5, COUP-TFI is expressed in the subpallium, and in the lateral ganglionic eminence (LGE) and MGE, respectively (Armentano et al., 2007; Faedo et al., 2008) (Fig. 1A). However, at E13.5, COUP-TFI expression becomes highly regionalized with a low rostral to high caudal expression gradient (Fig. $1 B-D$ ). Whereas COUP-TFI expression is restricted to the corticostriatal boundary and dorsal MGE (dMGE) at rostromedial levels (Fig. 1B), its expression increases at intermediate levels and includes also the preoptic area (Fig. 1C) (Flames et al., 2007). Expression becomes highest in the caudalmost basal telencephalon, particularly in the ventricular zone (VZ) and subventricular zone (SVZ) of the ventral CGE (vCGE), whereas in the LGE and dorsal CGE (dCGE) expression of COUP-TFI is lower (Fig. 1D). Thus, we found that, at E13.5, COUP-TFI becomes restricted in a rostrocaudal and dorsoventral expression gradient in the basal telencephalon, being strongest in the VZ and SVZ of the ventrocaudal ganglionic eminence, the vCGE.

COUP-TFI is also expressed in immature interneurons migrating tangentially from the basal telencephalon to the developing neocortex, as previously described (Tripodi et al., 2004), and in mature cortical interneuron subtypes at postnatal stages (this study) (Fig. $\left.1 E-J^{\prime \prime \prime}\right)$. Double labeling for COUP-TFI protein and the glutamate decarboxylase GAD67 transcript shows a high proportion of postnatal GABAergic interneurons positive for COUP-TFI in the somatosensory cortex $(83.1 \pm 2.2 \% ; n=3$; P8) (Fig. $1 E, E^{\prime}$ ). To further address whether specific subtypes of cortical interneurons express COUP-TFI, we performed double labeling for COUP-TFI and different molecular markers of mature cortical interneurons including the peptide hormone SST, NPY, and VIP at P8 $(n=3)$ (Fig. $\left.1 F-H^{\prime}\right)$, as well as the calciumbinding proteins $\mathrm{CR}$ and $\mathrm{PV}$ at $\mathrm{P} 21(n=3)$ (Fig. $\left.1 \mathrm{I}, J^{\prime \prime \prime}\right)$ within the somatosensory cortex. At P8, COUP-TFI was found in $55.8 \pm$ $1.1 \%$ of $S S T$-expressing, in $70.0 \pm 2.1 \%$ of NPY-expressing, and in $78.1 \pm 5.4 \%$ of VIP-expressing interneurons (Fig. $1 F-H^{\prime}$ ). At P21, we found only a $4.0 \pm 0.3 \%$ overlapping between COUPTFI and PV, and 51.6 $\pm 5.3 \%$ colabeling between COUP-TFI and CR (Fig. 1I, $\left.J^{\prime \prime \prime}\right)$. However, detailed morphological analysis indicates that COUP-TFI is highly expressed $(89.2 \pm 5.9 \%)$ in a subpopulation of CR + cells that shows a characteristic vertically oriented bipolar morphology (Fig. $\left.1 I^{\prime}-I^{\prime \prime \prime}\right)$. Together, these data indicate that, at postnatal stages, COUP-TFI is expressed in most mature cortical interneuron subtypes, with a preference to CGE-derived interneurons, such as bipolar VIP + and CR + cells, and to a subpopulation of NPY + cells (Karagiannis et al., 2009), whereas it is generally excluded from the MGE-derived PV-expressing cells.

\section{Specific inactivation of COUP-TFI in SVZ intermediate progenitors of the basal telencephalon}

To investigate the role of COUP-TFI in interneuron diversity and in the maturation and specification of different cortical interneuron subtypes, we used a conditional genetic approach in which the COUP-TFI lox/lox (COUP-TFF flox ) line (Armentano et al., 


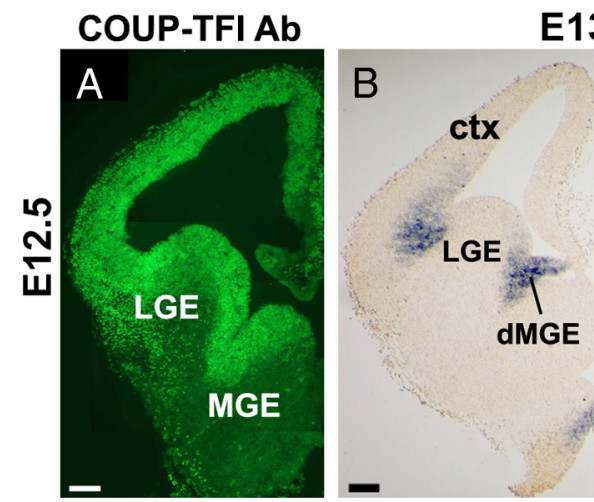

E13.5 COUP-TFI mRNA
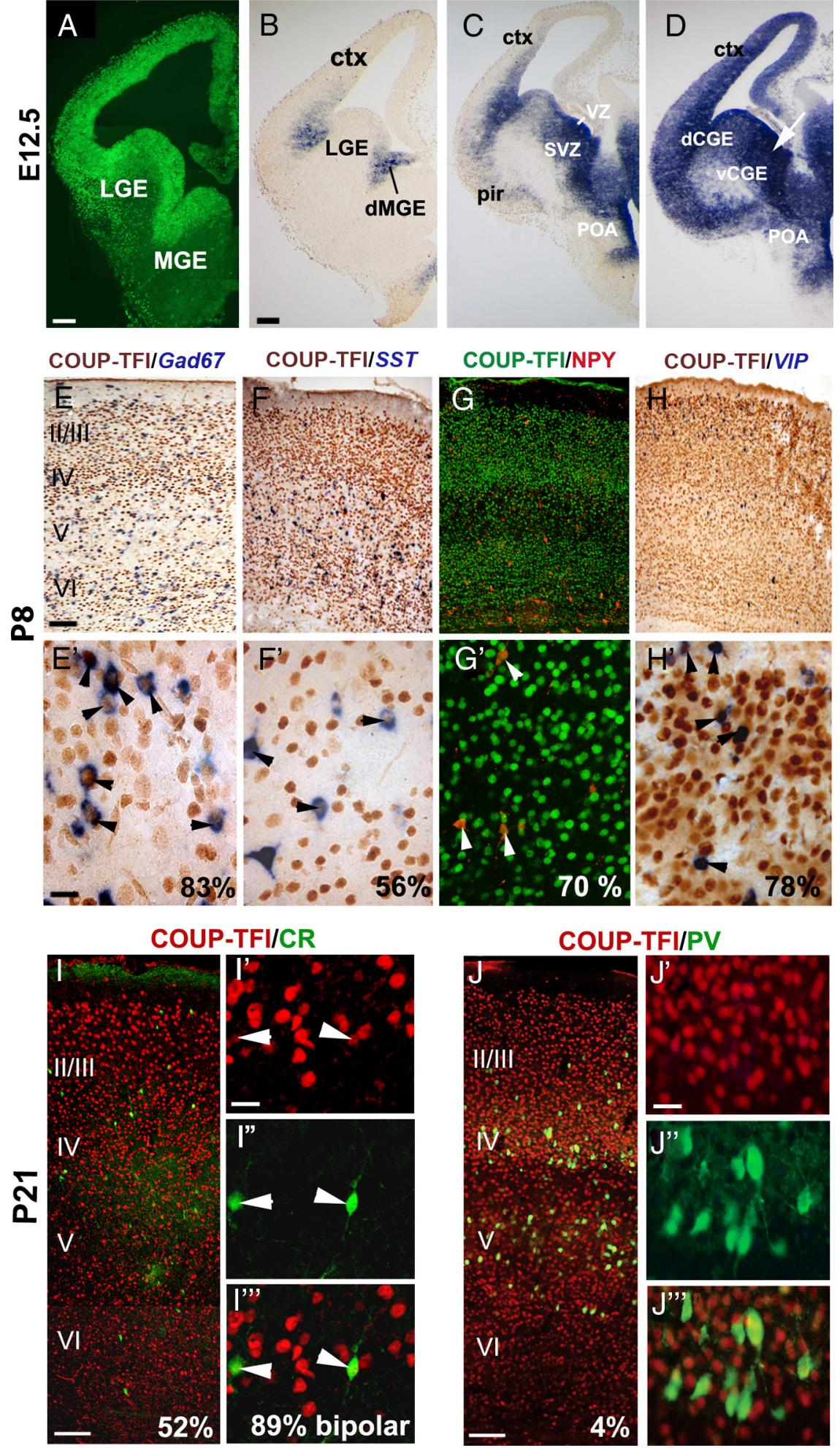

Figure 1. COUP-TFI becomes gradually restricted to the dorsal MGE and CGE and is highly expressed in CGE-derived cortical interneurons. $\boldsymbol{A}$, Coronal section of E12.5 wild-type embryo at intermediate levels showing localization of COUP-TFI protein in the telencephalon. Note high expression in the LGE and dorsal half of the MGE. $\boldsymbol{B}-\boldsymbol{D}$, Rostral to caudal sequential coronal sections of E13.5 wild-type embryos hybridized with COUP-TFI riboprobe indicate a low rostral to high caudal expression gradient, with regionalized expression in the dMGE $(\boldsymbol{B}, \boldsymbol{C})$ and preoptic area $(P O A)(\boldsymbol{C}, \boldsymbol{D})$. Expression is confined to the VZ and SVZ. The arrow in $\boldsymbol{D}$ indicates highest COUP-TFI expression levels in vCGE. $\boldsymbol{E}$ - $\boldsymbol{H}$, Double immunostaining for COUP-TFI protein and in situ hybridization for Gad67, SST, and $\operatorname{VIP}(\boldsymbol{E}, \boldsymbol{F}, \boldsymbol{H})$ and double immunofluorescence for COUP-TFI and NPY in P8 somatosensory cortices $(\boldsymbol{G})$. The roman numerals denote cortical layers. $\boldsymbol{E}^{\prime}-\boldsymbol{H}^{\prime}$, High-magnification views of a representative detail from $\boldsymbol{E}-\boldsymbol{H}$ indicate double-positive cells (arrowheads) and the different percentage of Gad67+ (83\%), SST + (56\%), NPY + (70\%), and VIP + (78\%) interneurons expressing COUP-TFI. $\boldsymbol{I}-\boldsymbol{J}^{\prime \prime \prime}$, Double immunofluorescence for COUP-TFI and CR $(\boldsymbol{I})$, and COUP-TFI and PV in P21 somatosensory cortices $(\boldsymbol{J})$. High-
2007) was mated to the Dlx5/6-Cre-IRESGFP transgenic line (Stenman et al., 2003), which drives the CRE-mediated activity exclusively within the basal telencephalic SVZ and mantle zone (MZ) (Fig. 2A). Mice homozygous for COUP-TFI flox and heterozygous for Dlx5/6-Cre-IRES-GFP are viable and fertile, and will be named COUP-TFI CKO-Dl $x 5 / 6$ throughout this study.

We first assessed whether and at what age COUP-TFI was specifically inactivated in Dlx5/6-positive cells by taking advantage of the endogenous GFP fluorescence of the Dlx5/6-Cre-IRES-GFP transgenic line (Stenman et al., 2003). In the basal telencephalon of COUP-TFI CKO-Dlx5/6 heterozygotes, only SVZ progenitors are double positive for GFP and COUP-TFI (Fig. 2E, $H$ ), whereas VZ progenitors express COUP-TFI (in red) but not GFP (in green) (Fig. 2C, F, D, G). No, or very few, COUP-TFI/GFP doublepositive cells are detected in the SVZ or mantle zone of COUP-TFI CKO-Dlx5/6 homozygous embryos at E12.5 and E13.5 (Fig. $2 E^{\prime}, H^{\prime}$ ) (data not shown), indicating that, already at E12.5, before COUP-TFI becomes regionalized to $\mathrm{dMGE}$ and CGE, COUP-TFI function is specifically abolished in the SVZ (Dlx5/6-positive domain), but not in progenitors located in the VZ (Dlx5/6-negative domain). Accordingly, at E15.5, COUP-TFI fails to be expressed in postmitotic neurons of the basal telencephalon (Fig. $2 I-J^{\prime}$ ), with the exception of the ventrally migrating stream originating from the Dlx5/6-negative interganglionic region, which coexpress COUPTFI and COUP-TFII (Tripodi et al., 2004). Thus, we generated a conditional COUPTFI mutant line, in which COUP-TFI is specifically inactivated in SVZ progenitors and postmitotic interneurons, but not in $\mathrm{VZ}$ progenitors.

Conditional loss of COUP-TFI function alters the balance of interneuron subtypes without affecting the total number of GABAergic cortical interneurons

Next, we investigated whether loss of function of COUP-TFI in SVZ progenitors of the basal telencephalon would affect the total number and laminar distribution of cortical interneurons at P21 in

magnification views indicate highest colocalization of COUPTFI with bipolar (R-positive cells ( $I^{\prime}-I^{\prime \prime \prime}$, arrowheads), and lowest colocalization of COUP-TFI with PV-positive cells $\left(J^{\prime}\right.$ $\left.J^{\prime \prime \prime}\right)$. Abbrevations: ctx, Cortex; dMGE, dorsal medial ganglionic eminence; LGE, lateral ganglionic eminence; pir, piriform cortex. Scale bars: $\boldsymbol{A}-\boldsymbol{D}, 200 \mu \mathrm{m} ; \boldsymbol{E}-\boldsymbol{J}, 100 \mu \mathrm{m} ; \boldsymbol{E}^{\prime}-\boldsymbol{J}^{\prime \prime \prime}, 50 \mu \mathrm{m}$. 
A

COUP-TFI pan-GABAergic conditional KO (COUP-TFI CKO-DIX5/6)

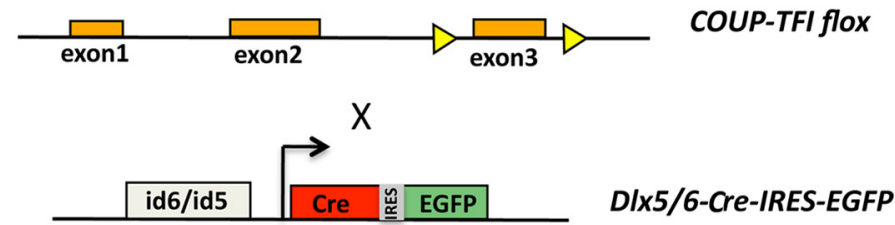

CKO DIX5/6 hom
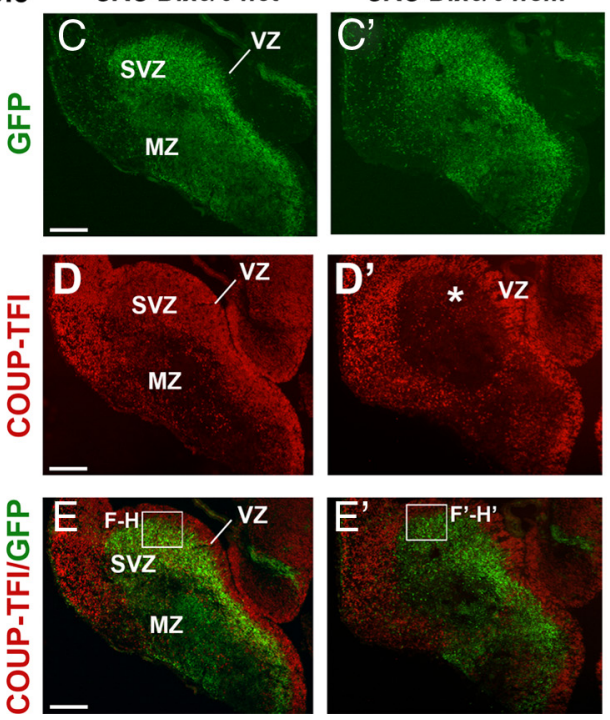
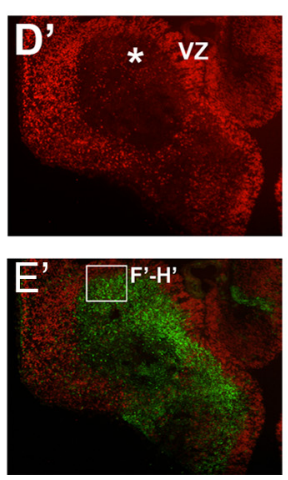

CKO D/x5/6 het
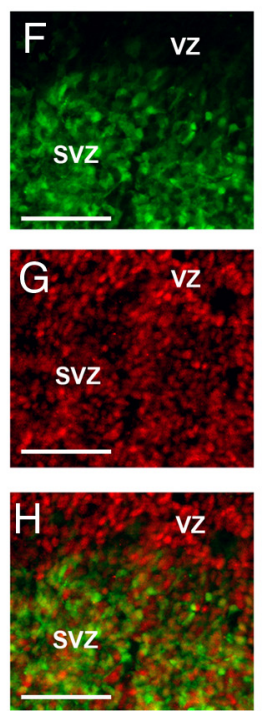

CKO DIx5/6 hom
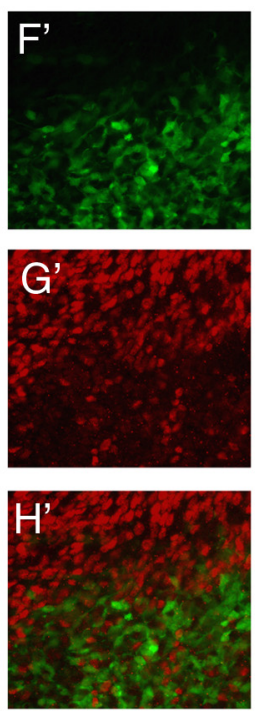

B

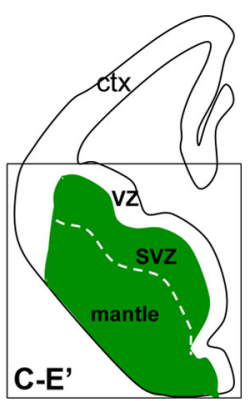

E15.5

COUP-TFI
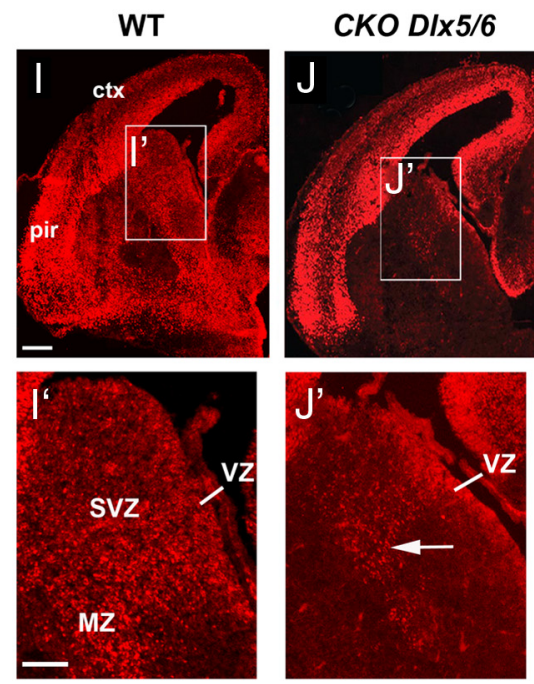

Figure 2. Conditional loss of COUP-TFl in cortical interneurons affects SVZ progenitors in the basal telencephalon. $A$, Schematic of the genetic strategy for examining COUP-TFI conditional loss of function by using the Dlx5/6-Cre-IRES-EGFP mouse active in the SVZ progenitors and postmitotic interneurons of the basal telencephalon (Stenman et al., 2003). B, Schematic of a E13.5 coronal hemisection indicating the position of $C-\boldsymbol{E}^{\prime}$. Green indicates the expression of the EGFP on Cre-mediated recombination. $\mathbf{C}-\boldsymbol{E}^{\prime}$, Coronal sections of E13.5 COUP-TFI CKO-DIx5/6 (CKO-DIx5/6) heterozygotes and homozygotes immunostained with anti-GFP $\left(\boldsymbol{C}, \boldsymbol{C}^{\prime}, \boldsymbol{F}, \boldsymbol{F}^{\prime}\right)$, anti-COUP-TFI $\left(\boldsymbol{D}, \boldsymbol{D}^{\prime}, \mathbf{G}, \boldsymbol{G}^{\prime}\right)$, and merge sections $\left(\boldsymbol{E}, \boldsymbol{E}^{\prime}, \boldsymbol{H}, \boldsymbol{H}^{\prime}\right)$. Note that GFP is expressed in the SVZ and MZ, but absent in the VZ, whereas COUP-TFI is expressed in the VZ, SVZ, and MZ of the basal telencephalon. $\boldsymbol{F}-\boldsymbol{H}^{\prime}$, High-magnification views of a region in the basal telencephalon indicated in $\boldsymbol{E}$ and $\boldsymbol{E}^{\prime}$. $\boldsymbol{E}^{\prime}, \boldsymbol{H}^{\prime}$, In COUP-TFI CKO-DIX5/6 homozygotes, no double-labeled cells are detected, confirming loss of COUP-TFI function in SVZ and MZ (DIx5/6-positive domains), but not in progenitors located in VZ (DIx5/6-negative domain). I-J', Immunostaining of anti-COUP-TFI in E15.5 WT and CKO-DIx5/6 embryos confirms specific inactivation of COUP-TFI in the SVZ and MZ of the basal telencephalon. I' $J^{\prime}$, High-magnification views of a region in the basal telencephalon indicated in $I$ and $J$. Note that expression of COUP-TFI in the VZ and in the ventrally migrating stream is not affected in COUP-TFI conditional mutants ( $\boldsymbol{J}^{\prime}$, arrow). Scale bars: $\left(-\boldsymbol{E}^{\prime}, \boldsymbol{I}, \boldsymbol{J}, 200 \mu \mathrm{m} ; \boldsymbol{F}-\boldsymbol{H}^{\prime}, 50 \mu \mathrm{m} ; \boldsymbol{I}^{\prime}, \boldsymbol{J}^{\prime}, 100 \mu \mathrm{m}\right.$.

COUP-TFI CKO-Dlx5/6 adult brains compared with control brains. Interestingly, we found no difference in the overall number of cortical interneurons between COUP-TFI mutant and WT sensorimotor cortices (Fig. $\left.3 A, A^{\prime}, G\right)(p=0.79 ; n=3)$, and no difference in the radial distribution of GABA-positive cells along the cortical wall of the COUP-TFI CKO-Dlx5/6 compared with WT (Fig. $3 H$ ). We next evaluated the total number and the radial distribution of distinct cortical interneuron subtypes by performing immunostaining and in situ hybridization using subtype-defining molecular markers for distinct GABAergic interneuron subpopulations (Ascoli et al., 2008) in P21 WT and COUP-TFI CKO-Dl $x 5 / 6$ cortices (Fig. $3 B-F^{\prime}$ ). As previously reported, $\mathrm{PV}+$ and SST + interneurons are two MGE early-born subclasses predominantly located in deep layers, whereas VIP+ cells are primarily CGE late-born interneurons located in superficial layers (Kawaguchi and Kubota, 1997; Butt et al., 2005; Wonders and Anderson, 2006; Miyoshi et al., 2007, 2010). By contrast, $\mathrm{CR}+$ and NPY + interneurons derive from both the MGE and the CGE, and the preoptic area, and are distributed along the radial extent of the cortex, although bipolar CR + cells are predominantly located in superficial layers (Xu et al., 2004; Fogarty et al., 2007; Caputi et al., 2009).
Interestingly, we found that loss of COUP-TFI function affects the specification of PV + interneurons and the VIP + and $\mathrm{CR}+$ bipolar subpopulations in opposite ways (Fig. 3I). Whereas the number of PV + interneurons was increased significantly by $33 \%$ (Fig. 3B, $\left.B^{\prime}, I\right)(p=0.0004 ; n=3)$, the total number of bipolar CR+ and VIP+ interneurons was decreased significantly by $21 \%$ (Fig. $\left.3 E, E^{\prime}, I\right)(p=0.007 ; n=3$ ) and by $26 \%$ (Fig. $\left.4 F, F^{\prime}, I\right)(p=0.03 ; n=3)$, respectively. Conversely, there was no significant change in the total number of SST + (Fig. $3 C, C^{\prime}, I$ ) $(p=0.09 ; n=3)$ and NPY $+\left(\right.$ Fig. $\left.3 D, D^{\prime}, I\right)(p=0.19 ; n=3)$ subpopulations, although the number of NPY + interneurons increased by $25 \%$ in deep layers of COUP-TFI CKO-Dlx5/6 cortices (Fig. $3 J)(p=0.0028 ; n=3)$. Differences in layer distribution were also detected in the MGE-derived $\mathrm{PV}+$ population, which was strikingly increased by $>50 \%$ in deep layers (Fig. $3 J$ ) $(p<0.0001 ; n=3)$, but not affected in superficial layers (Fig. $3 K)(p=0.98)$ of COUP-TFI CKO-Dlx5/6 mice. Regarding the CGE-derived subpopulations, the number of VIP-expressing cells was reduced significantly in all layers (36\% reduction in deep layers, $p=0.02$; and $22 \%$ in superficial layers, $p=0.04 ; n=3$ ) (Fig. $3 F, F^{\prime}, I$ ), whereas the number of CR-expressing cells was decreased by $27 \%$ in superficial layers (Fig. $3 K)(p=0.0034$; 


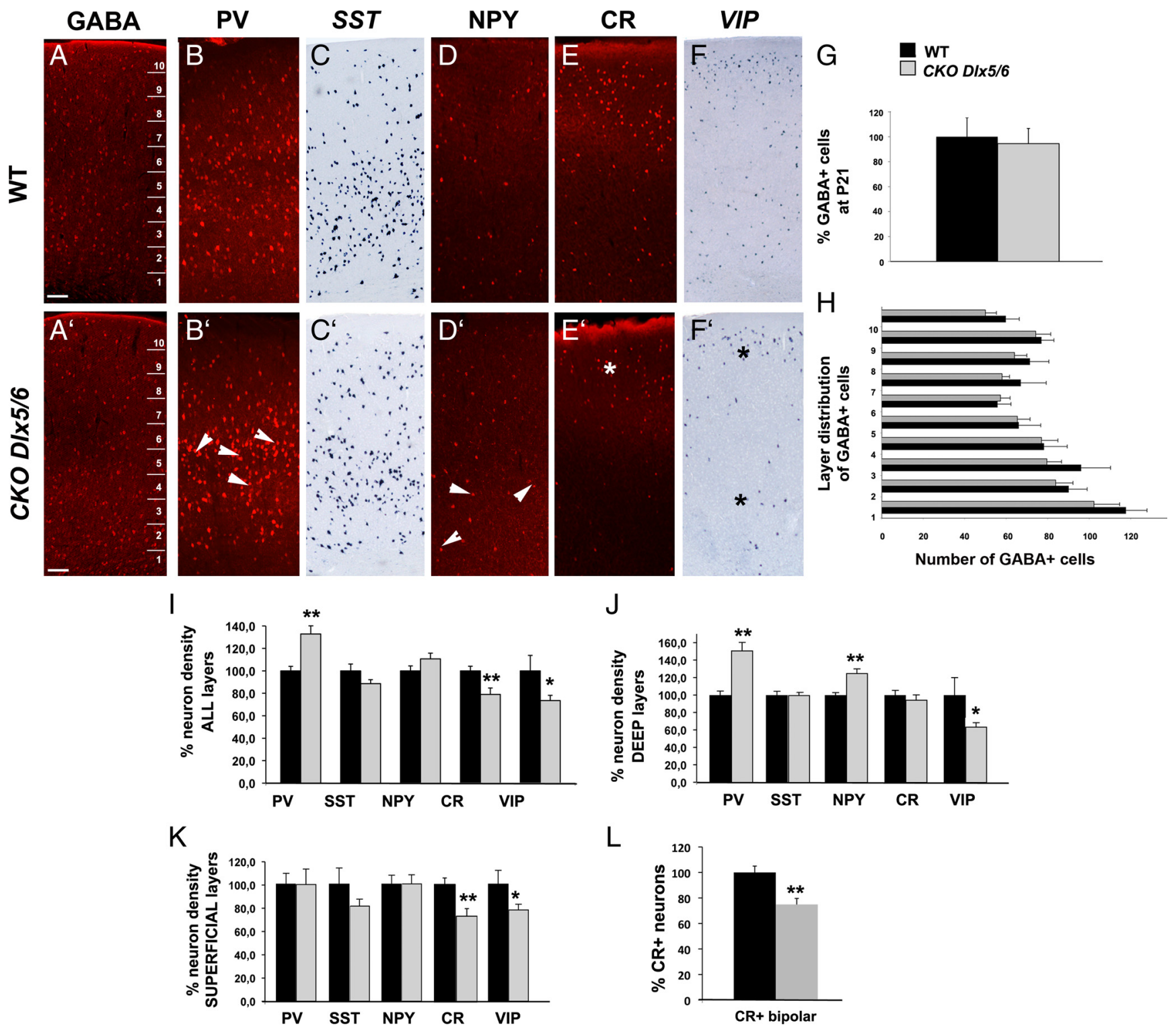

Figure 3. Altered balance between PV- and bipolar VIP- and CR-expressing cortical interneurons in the absence of COUP-TFI function. Representative coronal sections of P21 WT and CKO-Dlx5/6 cortices within the P21 sensorimotor cortex of WT $(\boldsymbol{A}-\boldsymbol{F})$ and $C K O-D / \times 5 / 6(C O U P-T F I C K O-D / \times 5 / 6)\left(\boldsymbol{A}^{\prime}-\boldsymbol{F}^{\prime}\right)$ immunostained with anti-GABA $\left(\boldsymbol{A}-\boldsymbol{A}^{\prime}\right)$ and with different cortical interneuron subtypes, as indicated $\left(\boldsymbol{B}-\boldsymbol{F}^{\prime}\right)$. $C, C^{\prime}, F, F^{\prime}$, In situ hybridization of SST and VIP on adjacent sections. $\mathbf{G}, \boldsymbol{H}$, The number and distribution of GABA + cells were quantified by subdividing the cortex into 10 bins along the ventricular (bin 1 ) to pial (bin 10) axis of equivalent areas of the sensorimotor cortex from WT and $C K 0-D / x 5 / 6$ animals. No significative difference in the total amount of GABA + cells $(\boldsymbol{G})$ and in the distribution of cells in each bin $(\boldsymbol{H})$ was detected between WT and mutant cortices. The arrowheads in $\boldsymbol{B}^{\prime}$ and $\boldsymbol{D}^{\prime}$ point to an increased number of PV- and NPY-expressing cells, respectively, in deep layers. The asterisk in $\boldsymbol{E}^{\prime}$ denotes a reduction of CR-expressing cells in superficial layers, whereas asterisks in $\boldsymbol{F}^{\prime}$ indicate a reduction of VIP-expressing cells in both superficial and deep layers. $I-L$, Graphical representation of the percentage of density of the different cortical interneuron subpopulations in the COUP-TFICKO relative to WT, as indicated. I, Along the whole radial extent of the cortex, the number of the PV + subpopulation increased significantly by $33 \%$ (WT, 165.3 $\pm 6.7 ;(K 0,219 \pm 12.4 ; p<0.01 ; n=3)$, whereas the total number of bipolar VIP + and CR + interneurons decreased significantly by 26\% (VIP; WT, 208.2 $\pm 28.6 ; C K 0,153.1 \pm 9.8 ; p=0.03$; $n=3)$ and by $21 \%(C R ; W T, 130.9 \pm 5.4 ;(K 0,103.5 \pm 7.1 ; p=0.01 ; n=3)$, respectively.J, In deep layers, thePV+ (WT,85.9 $\pm 4.5 ; C K 0,130.1 \pm 8.4 ; p<0.001 ; n=3)$ and NPY + (WT, 42.8 $\pm 1.4 ;(K 0$, $53.71 \pm 2.3 ; p=0.003 ; n=3$ ) cohorts increased significantly, whereas the VIP + subpopulation decreased significantly (WT, 64.1 $\pm 13.3 ;$ CKO, $40.8 \pm 3.3 ; p=0.02 ; n=3$ ) in $C K 0-D / x 5 / 6$ mutants. $K$, In superficial layers, the CR + (WT, $94.7 \pm 5.1 ;$ CKO, 69.2 $\pm 5.8 ; p=0.003 ; n=3)$ and VIP $+($ WT, $144.1 \pm 16.8 ;(K 0,112.3 \pm 6.9 ; p=0.04 ; n=3)$ subpopulations are decreased significantly in CKO-Dlx5/6 mice. $L$, Graphical representation of the percentage of bipolar-shaped CR+ interneurons indicates a statistically significant decrease of $25 \%$ in $C K 0-D / x 5 / 6$ cortices (WT, 21.8 $\pm 1.2 ;$; CKO, 16.3 $\pm 1.4 ; p=0.008$; $n=3)$. Scale bars: $\boldsymbol{A}-\boldsymbol{F}^{\prime}, 100 \mu \mathrm{m}$. Error bars indicate SEM.

$n=3)$, but not altered in deep layers (Fig. $3 J)(p=0.57 ; n=3)$. Detailed morphological characterization of the $\mathrm{CR}+$ subpopulation demonstrated a $25 \%$ decrease (Fig. $3 L)(p=0.007 ; n=3)$ of bipolar-shaped cells, strongly indicating that the $\mathrm{CR}+$ cohort altered in COUP-TFI CKO-Dlx5/6 mice was mainly composed of bipolar CR + cells (Caputi et al., 2009). Together, these results indicate that loss of COUP-TFI function alters the balance between PV+ (MGE-derived) and bipolar VIP+, CR+ (CGEderived) interneuron subpopulations, without affecting the overall cortical interneuron number.
COUP-TFI does not act on cell identity but regulates cell cycle divisions of SVZ precursors in the basal telencephalon

An increase of MGE-derived PV + interneurons at the expense of CGE-derived CR + and VIP+ interneurons might suggest either a molecular switch between MGE and CGE identity or an imbalance in the rate of proliferation/differentiation between MGE- and CGEderived cortical interneurons. To discern between these two hypotheses, we first investigated whether developmental regulators of MGE interneuron cell fate were abnormally expressed in COUP-TFI CKO-Dlx $5 / 6$ embryos. We looked at the expression of $N k x 2.1$, 
known to determine the identity of progenitor cells in the MGE (Sussel et al., 1999; Butt et al., 2008; Nóbrega-Pereira et al., 2008), and its downstream target Lhx6 (Du et al., 2008), which starts to be expressed as soon as MGE-derived progenitors leave the $\mathrm{VZ}$ (Liodis et al., 2007). No obvious changes in the expression pattern of $N k \times 2.1$ and Lhx6 at rostromedial and caudal levels were observed in E13.5 COUP-TFI CKO-Dlx5/6 embryos (Fig. $4 A-D^{\prime}$ ), indicating that loss of COUP-TFI in the SVZ does not induce a molecular fate change between CGE- and MGEderived interneurons.

To further assess whether inactivation of COUP-TFI would have an effect on cell cycle divisions, similarly to what observed in the neocortex (Faedo et al., 2008; Tomassy et al., 2010), we performed acute injection of BrdU in pregnant females and compared the percentage of BrdUpositive cells in the MGE and CGE of WT and E13.5 COUP-TFI CKO-Dlx5/6 embryos (Fig. $4 E-F^{\prime}$ ). Interestingly, the ratio of proliferating $(\mathrm{BrdU}+)$ cells was significantly higher in the MGE, and tends to increase in the CGE without, however, reaching statistical significance (Fig. 4G) [WT: MGE, $25.7 \pm 2.2 \%$; CGE, $30.2 \pm$ 2.0\%; CKO: MGE, $47.8 \pm 3.9 \%$; CGE, $45.0 \pm 4.2 \% ; p(\mathrm{MGE})=0.040 ; p(\mathrm{CGE})=$ 0.097]. Similar results were obtained after immunofluorescence of the proliferation marker Ki67, in which the thickness of the Ki67-positive region, relative to the total ventricular-to-pial thickness of the basal telencephalon, results larger in the MGE than CGE of mutant embryos (Fig. 4H,I) [WT: MGE, $34.2 \pm 4.1 \%$; CGE, $27.0 \pm$ 2.0\%; CKO: MGE, $47.3 \pm 0.4 \%$; CGE, $33.8 \pm 1.8 \% ; p(\mathrm{MGE})=0.033 ; p(\mathrm{CGE})=$ $0.062]$. Thus, increased proliferation, particularly in the MGE region, was detected in COUP-TFI CKO-Dlx5/6 embryos. To further investigate whether cell cycle progression was affected in the absence of COUP-TFI in cortical interneurons, we assessed expression of the $\mathrm{G}_{1}$-active cell cycle marker cyclinD2, known to promote SVZ divisions in the telencephalon (Glickstein et al., 2007b). Strikingly, we found that expression of cyclinD2 was increased in SVZ progenitors of E13.5 and E15.5 COUP-TFI CKO-Dlx5/6 embryos, predominantly at rostromedial levels (Fig. $\left.4 J-K^{\prime}\right)$. Since COUP-TFI is maintained in $\mathrm{VZ}$ progenitors of mutant embryos, we found accordingly no differences in the expression of cyclinD1 (data not shown) (Glickstein et al., 2007a). Together, these data strongly suggest that COUP-TFI regulates cell cycle progression by modulating
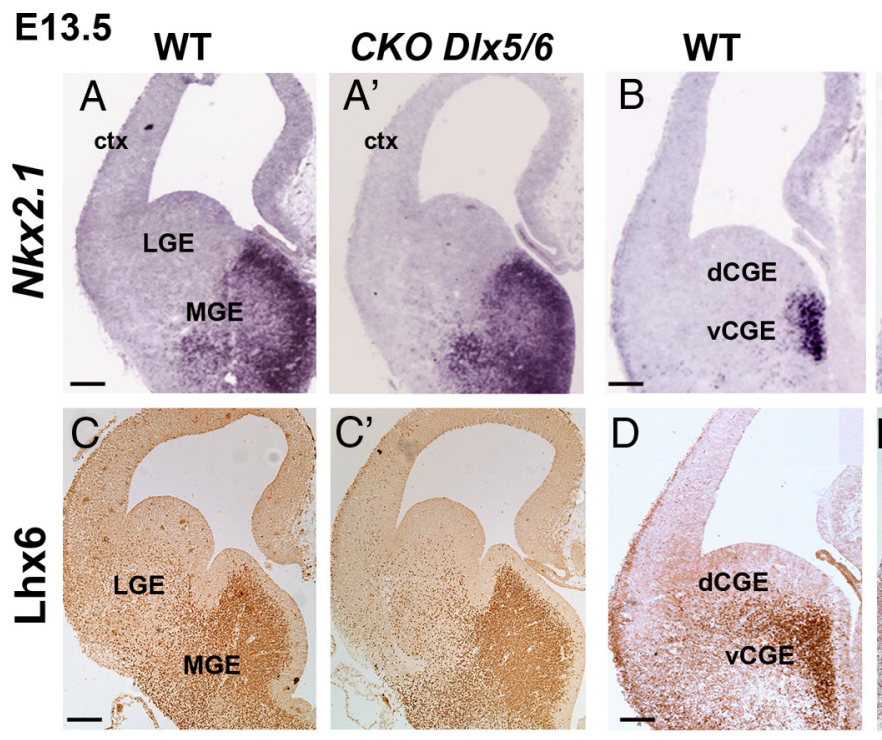

CKO DIX5/6
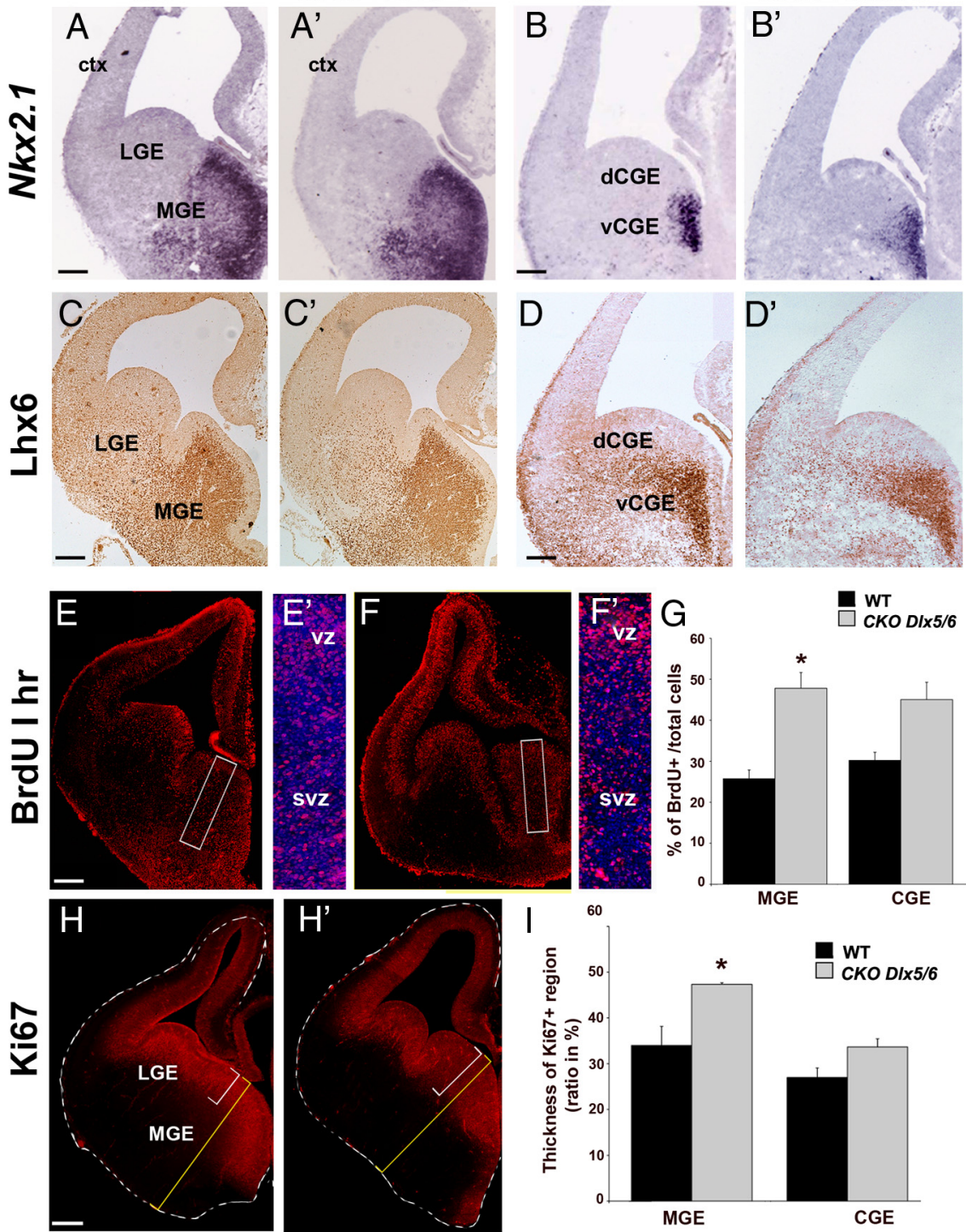

E13.5
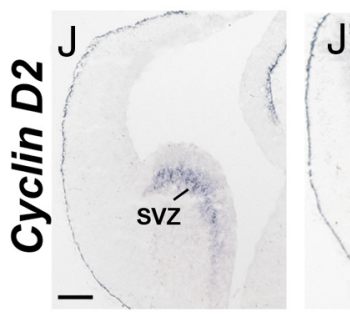

\section{E15.5}

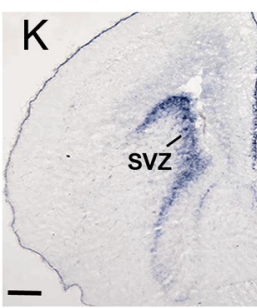

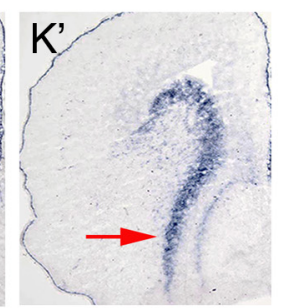

Figure 4. Normal molecular identity but increased rates of proliferation in COUP-TFI conditional mutants. $\boldsymbol{A}-\boldsymbol{B}^{\prime}, \boldsymbol{C}-\boldsymbol{D}^{\prime}$, In situ hybridization of Nkx2.1 $\left(\boldsymbol{A}-\boldsymbol{B}^{\prime}\right)$ and immunohistochemistry of Lhx6 $\left(\boldsymbol{C}-\boldsymbol{D}^{\prime}\right)$ on E13.5 coronal sections on intermediate $\left(\boldsymbol{A}, \boldsymbol{A}^{\prime}, \boldsymbol{C}_{,} \boldsymbol{C}^{\prime}\right)$ and caudal $\left(\boldsymbol{B}, \boldsymbol{B}^{\prime}, \boldsymbol{D}, \boldsymbol{D}^{\prime}\right)$ levels indicate no relevant changes in the expression profiles of $N k \times 2.1$ and $L \mathrm{~h} \times 6$ in $C K 0$-D/x5/6 embryos. $\boldsymbol{E}, \boldsymbol{F}$, BrdU immunofluorescence on DAPI-stained intermediate coronal sections indicate increased proliferation in the MGE of E13.5 CKO-D/x5/6 embryos. $\boldsymbol{E}^{\prime}, \boldsymbol{F}^{\prime}$, High-magnification views on the boxes depicted on $\boldsymbol{E}$ and $\boldsymbol{F}$. $\boldsymbol{G}$, Graphical representation demonstrates a statistically significative increase of BrdU-positive cells in the MGE $\left({ }^{*} p=\right.$ $0.040)$, but not CGE ( $p=0.097)$, although the percentage of BrdU-positive cells tends to increase in the CGE. $\boldsymbol{H}, \boldsymbol{H}^{\prime}, \mathrm{Ki} 67$ immunofluorescence labels highly proliferating cells in intermediate coronal sections of E13.5 of WT and CKO-D/X5/6 embryos. I, Graphical representation indicates a statistically significative increase in the size of the Ki67-positive region of the MGE (white brackets) relative to the total ventricular-to-pial thickness of mutant embryos (yellow brackets) $\left({ }^{*} p=\right.$ 0.03). Error bars indicate SEM. $\boldsymbol{J}^{\prime}-\boldsymbol{K}^{\prime}$, Expression of the cell cycle protein cyclinD2 is upregulated in the SVZ of E13.5 ( $\boldsymbol{J}^{\prime}$, arrow) and E15.5 (KO-D/x5/6 embryos ( $\boldsymbol{K}^{\prime}$, arrow). Abbrevations: dCGE, Dorsal caudal ganglionic eminence; vCGE, ventral caudal ganglionic eminence; ctx, cortex. Scale bars, $200 \mu \mathrm{m}$. 

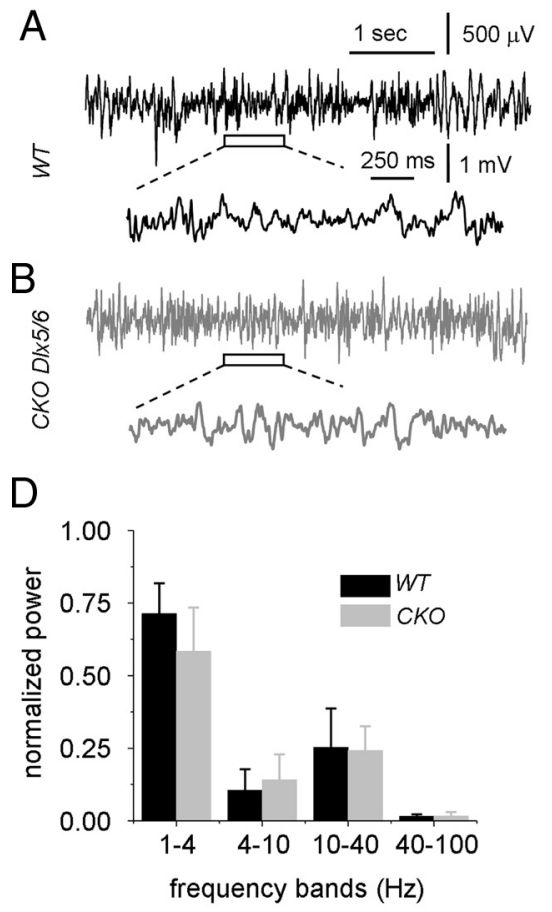

Figure 5. Normal basal EEG recordings in COUP-TFI conditional mutants. $A$, Cortical EEG recorded from awake WT mice shows normal activity at $3-7 \mathrm{~Hz}$ with episodes of beta activity at $15-20 \mathrm{~Hz}$. Higher resolution trace shows details from the upper traces. $B$, EEG recordings from awake COUP-TFI CKO-D/x5/6 mice exhibit approximately similar features than WT animals. C, Normalized power spectra from the representative examples shown in $\boldsymbol{A}$ and $\boldsymbol{B}$ (left) and for the population means (right). No differences were found between groups. A 95\% confidence interval is represented with dotted lines for each group. $\boldsymbol{D}$, Mean spectral power per frequency bands was not different between groups. Error bars indicate SEM. $\boldsymbol{E}$, Age dependence of the spectral content at the different frequency bands.

divisions of SVZ interneuron precursors without affecting the identity of MGE determinant genes.

\section{Basal EEG recordings show no apparent abnormalities in mutant mice}

Previous reports have shown that altered balance of interneuronal subtypes correlate with epileptic phenotypes and disruption of cortical EEG rhythms (Powell et al., 2003; Cobos et al., 2005; Glickstein et al., 2007b; Butt et al., 2008; Batista-Brito et al., 2009). We therefore chose to obtain long-lasting recordings of basal EEG activity from adult WT $(n=6)$ and COUP-TFI CKODlx5/6 mice $(n=8)$. Cortical EEGs from awake WT mice were characterized by rhythmic activity at 3-7 Hz interspersed by episodes of beta activity at 15-20 Hz (Fig. 5A). Strikingly, COUPTFI CKO-Dlx5/6 mice showed approximately similar EEG patterns compared with WT (Fig. 5B). Seizures, and other types of epileptic-like activity, were never observed in mutants, and no statistical difference was evident from the normalized mean power spectra from both groups (Fig. $5 C)(U=25 ; p>0.491)$. In a group of mice $(n=6 \mathrm{WT} ; n=10 \mathrm{CKO})$, we obtained simultaneous cortical and hippocampal EEG recordings to check for signs of ictal activity confined to the hippocampus with little cortical manifestation. Again, we did not observe signs of hyperexcitability in the hippocampus of COUP-TFI CKO-Dl $x 5 / 6$ mice (data not shown), discarding the possibility of nonconvulsive subclinical seizures in these animals.

We also looked for differences in the typical frequency bands of the cortical EEG spectrum [i.e., 1-4 Hz (delta), 4-10 Hz (theta), $10-40 \mathrm{~Hz}$ (beta), and $40-100 \mathrm{~Hz}$ (gamma)] (Fig. 5D). We found no difference between groups using a two-way
ANOVA comparison for the delta $(F=$ $1.45 ; p=0.25)$, theta $(F=0.78 ; p=0.39)$, and beta bands $(F=0.26 ; p=0.62)$, and the Kruskal-Wallis test for the gamma band $(U=25 ; p=0.49)$. Finally, we checked for a possible functional compensation in COUP-TFI mutants, as reflected in an age dependence of the cortical EEG activity. We found no correlation with age of the cortical EEG power in the different frequency bands over the course of 40 to $\sim 90 \mathrm{~d}$ postnatal (Fig. $5 E)$. Furthermore, no gender differences were present in the spectra $(U=19.5 ; p>$ $0.203)$. Thus, alterations in the distribution of PV,$+ \mathrm{VIP}+$, and CR + interneuron subpopulations result in no obvious abnormalities in the gross cortical EEG of COUP-TFI mutant mice.

\section{COUP-TFI mutants are more resistant to seizures induced by lithium- pilocarpine}

The preceding data suggest that reduced number of VIP + and CR + cortical interneurons has no discernible effect on the basal cortical activity of COUP-TFI mutants. This was surprising because a similar reduction in the presence of normal numbers of $\mathrm{PV}+$ interneurons results in dysrhythmia and epilepsy (Cobos et al., 2005). We thus reasoned that the increase of $\mathrm{PV}+$ and possibly NPY + interneurons observed in our mutants might be functionally compensating the reduction of CGE-derived interneuronal subtypes.

To test this hypothesis, we used the muscarinic receptor agonist pilocarpine to unspecifically increase the excitatory level at doses of $100 \mathrm{mg} / \mathrm{kg}$ that induce tonic-clonic seizures and status epilepticus in wild-type mice (see Materials and Methods) (Gröticke et al., 2007). We found that COUP-TFI CKO-Dlx5/6 mice $(n=7)$ were more resistant to seizures and to developing status epilepticus than WT $(n=7)$, as reflected in both the cortical EEG recordings and clinical manifestations (Fig. 6A,B). Progression to the status was characterized by the early appearance of body and tail rigidity, and multiple spikes in the cortical EEG between 2 and 3 min after pilocarpine injection (Fig. 6A,B). There was no significant difference between groups in the latency to the first EEG spikes (Fig. 6C). This was followed by whole-body clonic spasms and secondary generalized tonic-clonic seizures with forelimb automatisms, which were associated with ictal EEG patterns. The mean latency to the first electroclinical manifestation of a tonic-clonic seizure was $39.9 \pm 6.9 \mathrm{~min}$ in mutants versus $14.9 \pm 1.6 \mathrm{~min}$ in WT animals (Fig. $6 C)\left(F_{(1,12)}=12.48\right.$; $p=0.004)$. Similarly, the onset of the status epilepticus, defined from EEG recordings (Fig. $6 A, B$ ), was significantly delayed in COUP-TFI CKO-Dlx5/6 versus WT mice (Fig. 6C) (CKO, $50.4 \pm$ $6.9 \mathrm{~min}$, vs WT, $\left.22.8 \pm 3.3 \mathrm{~min} ; F_{(1,12)}=13.17 ; p=0.003\right)$. Together, these data indicate that COUP-TFI CKO-Dlx5/6 mice are more resistant to seizures induced by pilocarpine, probably reflecting a functional effect of the increased number of PV-, and eventually, NPY-expressing interneurons in counteracting a decreased number of VIP- and CR-positive neurons. 
Seizure resistance in mutant mice is mediated by $\mathrm{GABA}_{\mathrm{A}}$ and $\mathrm{GABA}_{\mathrm{B}}$ signaling

To further confirm that the seizureresistant character of these mice was GABA dependent, we used PTZ at $90 \mathrm{mg} /$ $\mathrm{kg}, \mathrm{a} \mathrm{GABA}_{\mathrm{A}}$ receptor antagonist that is widely used for testing seizure susceptibility in mice (Powell et al., 2003). Similarly to pilocarpine, we found an early appearance of body and tail rigidity in both WT $(n=22)$ and COUP-TFI CKO-Dlx5/6 $(n=12)$ mice and a larger latency of COUP-TFI mutants to the first tonicclonic seizure (Fig. 6D) (WT, $24 \pm 2.7$ min, vs CKO, $38 \pm 3.3 \mathrm{~min} ; F_{(1,32)}=9.59$; $p=0.004)$.

Together, this indicates that COUPTFI CKO-Dlx5/6 brains are less excitable than WT brains to produce seizures induced by the cholinomimetic convulsant pilocarpine and the $\mathrm{GABA}_{\mathrm{A}}$ antagonist pentylenetetrazole. However, latency difference to tonic-clonic seizures can be accounted by other mechanisms, like a different excitability level at the glutamatergic circuits or residual presynaptic and postsynaptic $\mathrm{GABA}_{\mathrm{B}}$ receptor-mediated inhibition after PTZ injection. To ascertain that the seizure-resistant feature of these mice are indeed GABA dependent, we preinjected WT $(n=7)$ and mutant animals $(n=8)$ with the $\mathrm{GABA}_{\mathrm{B}}$ receptor antagonist CGP 35348 at the nonconvulsive dose of $136 \mathrm{mg} / \mathrm{kg}$ intraperitoneally (Karlsson et al., 1992) 30 min before PTZ (Fig. 6D). CGP pretreatment significantly [genotype $\left(F_{(1,45)}=1.96 ; p=0.168\right)$; pretreatment $\left(F_{(1,45)}=20.77 ; p<0.0001\right)$; genotype by pretreatment $\left.\left(F_{(1,45)}=6.93 ; p=0.01\right)\right]$ reduced the latency to the first tonic-clonic seizure in COUP-TFI CKO-Dlx5/6 mice (CGP 35348 vs vehicle pretreated, $p<0.0001)$ and eliminated latency difference with the WT group ( $p=0.38$ ) (Fig. $6 D$ ). Thus, these data indicate that blockage of $\mathrm{GABA}_{\mathrm{B}}$ receptor abolishes resistance to seizures induced by the $\mathrm{GABA}_{\mathrm{A}}$ receptor antagonist PTZ, and confirm that the seizureresistant phenotype observed in COUP-TFI CKO-Dlx5/6 mice is dependent on an altered balance of MGE- versus CGE-derived interneurons acting through both $\mathrm{GABA}_{\mathrm{A}}$ and $\mathrm{GABA}_{\mathrm{B}}$ receptors.

\section{Discussion}

In this study, we show that COUP-TFI regulates the balance between distinct cortical interneuron subtypes in a spatiotemporal controlled manner during corticogenesis, thereby enabling proper cortical microcircuitry and functional activity. Conditional inactivation of COUP-TFI solely in interneuron SVZ progenitors leads to a decreased number of CR- and VIP-bipolar GABAergic cells in superficial cortical layers and a concomitant increase of PV- and NPYexpressing interneurons in deep cortical layers of the mature cortex, without affecting the total number of GABAergic cortical interneurons. Physiologically, this alters the balance of both $\mathrm{GABA}_{\mathrm{A}}$ and $\mathrm{GABA}_{\mathrm{B}}$ receptor-mediated inhibition so that mutants become more resistant to pharmacologically induced seizures. To our knowledge, this study describes for the first time an epilepsy-resistant phenotype
WT

B
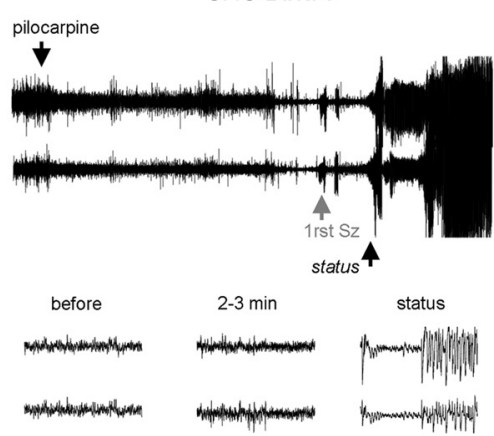

D

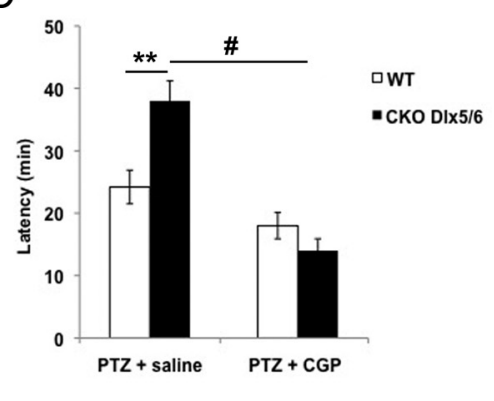

Figure 6. Seizure resistance of COUP-TFI CKO-D/x5/6 mice. A, Bilateral cortical EEG recordings obtained from a representative WT mouse during the progression to a status epilepticus induced by intraperitoneal injection of the muscarinic receptor agonist pilocarpine. The arrows indicate the timing of pilocarpine injections; the first generalized tonic-clonic seizure (first $\mathrm{Sz}$ ) and the 列 Expanded traces similar to that shown in $\boldsymbol{A}^{\prime}$ for COUP-TFI CKO-D/x5/6 (CKO-D/x5/6) mice. C, Mean latency to first spikes recorded antagonist PTZ. Pretreatment with the $G_{A B A_{B}}$ receptor antagonist CGP 35348 significantly reduces the latency to the first tonicconic seizures and eliminates latency difference between WT and mutants. ${ }^{* *} p<0.01$, CKO-DIx5/6 versus WT, within treatment; ${ }^{\#}<0.0001$, CGP 35348 versus saline pretreatment, within genotype. Error bars indicate SEM.

after genetic manipulation of interneuronal subtypes and suggests a potential role for increased PV + and NPY + interneurons in controlling seizures.

\section{COUP-TFI controls the balance between MGE- and CGE-derived interneurons}

Until recently, the CGE was not considered a distinct anatomical and molecular entity, mainly because of the absence of a morphologically definite sulcus demarcating the CGE from the MGE and LGE, and to the lack of CGE-restricted molecular markers (Flames et al., 2007). However, a series of experimental evidence including in vivo transplantation studies, in vitro migratory assays, and fate-mapping analyses have established the CGE as a separate molecular territory and confirmed that CGE-derived cortical interneurons contribute to a subset of interneuron subtypes with distinct morphological and electrophysiological interneurons (Nery et al., 2002; Yozu et al., 2005; Kanatani et al., 2008; Willi-Monnerat et al., 2008; Miyoshi et al., 2010). Still, no functional studies on genes required for the specification of CGE-derived cortical interneurons have been described to date.

Differently from COUP-TFI, COUP-TFI is expressed in precursors of the basal telencephalon along the whole rostrocaudal extent at early stages (Armentano et al., 2007; Faedo et al., 2008), before getting restricted to the dorsal MGE and CGE at E13.5, and to CGE-derived cortical interneurons, such as VIP- and 
CR-expressing bipolar interneurons ( $\sim 80$ and 90\%, respectively). Furthermore, $56 \%$ of COUP-TFI+ express SST, most presumably originating from the dMGE, whereas $70 \%$ express $\mathrm{NPY}+$, which might derive from the dMGE, the CGE, and, in a small contribution, the preoptic area (Fogarty et al., 2007; Gelman et al., 2009; Karagiannis et al., 2009; Sousa et al., 2009).

Interestingly, although COUP-TFI is expressed in the MGE at early stages during the production of PV + interneurons, its expression is not maintained in this cell type at P21. In this study, we demonstrate that loss of COUP-TFI function affects the generation, but not maturation of PV + interneurons. Differently from Nkx2.1, which normally acts as a cell fate switch between PV+ (MGE-derived) and CR+/VIP+ (CGE-derived) interneurons (Butt et al., 2008), COUP-TFI acts in the opposite way by limiting generation of $\mathrm{PV}+$ precursors and promoting specification of $\mathrm{CR}+$ and VIP + cells. However, the imbalance between MGEand CGE-derived interneurons induced by loss of COUP-TFI function is not attributable to a change of cell fate, as observed for Nkx2.1 mutants (Butt et al., 2008), but rather to a control on precursor cell divisions mainly within the MGE, as seen by increased proliferation and expression levels of the cell cycle protein cyclinD2 in SVZ precursors (Glickstein et al., 2007a,b). Null mutant mice for cyclinD2 have a selective deficit in cortical PVexpressing interneurons and increased excitability, without affecting other MGE-derived subtypes, such as SST + interneurons (Glickstein et al., 2007b). Accordingly, overexpression of $c y$ clinD2 in COUP-TFI conditional mutants results in an increase of PV + neurons (Glickstein et al., 2007b), implying that negative control of SVZ divisions by COUP-TFI normally limits the number of PV + interneurons. Thus, in the absence of COUP-TFI function, this repression is released and an excess of $\mathrm{PV}+$ interneurons is produced. Since COUP-TFI is expressed in the MGE from E10.5 to E12.5 (Armentano et al., 2006, 2007; this study), and production of PV + interneurons occurs from E9.5 to E15.5 (Miyoshi et al., 2007), it is reasonable to assume that presumptive PV+ cells express COUP-TFI while proliferating and that COUP-TFI normally controls early SVZ progenitor divisions during generation of $\mathrm{PV}+$ interneurons.

However, in addition to the increase of $\mathrm{PV}+$ interneurons, loss of COUP-TFI function also affects correct specification of late-born $\mathrm{CR}+$ and VIP + interneurons. We hypothesize that overproduction of PV + (and in part NPY + cells) at a time when normally VIP+ and CR + cells are generated (from E14.5 to E18.5) (Miyoshi et al., 2010), depletes the progenitor pool resulting in a decrease of VIP + and CR + bipolar interneuron precursors in the CGE. Thus, we propose that COUP-TFI normally regulates the number of $\mathrm{PV}+$ cells during generation of $\mathrm{CR}+$ and $\mathrm{VIP}+$ neurons by controlling sequential cell divisions in SVZ progenitors. Excessive cell divisions during the time of $\mathrm{PV}+$ interneuron generation would affect the sequential production of VIP/CR bipolar interneurons. In support of this mechanism, increased PV+ interneurons are found in deep cortical layers (appropriately for their MGE origin), and decreased CR + and VIP+ interneurons are still located in superficial layers (appropriate for their CGE origin) in COUP-TFI conditional mutants.

Together, our data support a model by which COUP-TFI regulates the fine balance between MGE- and CGE-derived interneurons by ensuring proper generation and specification of different subsets of cortical interneurons. This is in accordance with the control of pyramidal projection neurons by COUP-TFI, which is required to balance motor and sensory cortical areas by repressing corticospinal motor neuron generation during production of corticofugal pyramidal neurons in an area- and temporal-specific manner (Armentano et al., 2007; Tomassy et al., 2010). Moreover, both COUP-TFs regulate the switch between neurogenesis (early corticogenesis) and gliogenesis (late corticogenesis), and in their absence, neurogenesis is sustained and the generation of early-born neurons is prolonged (Naka et al., 2008). Finally, the Drosophila COUP-TF ortholog, SVP, controls neuroblast diversity in a temporally controlled mode by regulating the balance between early- and late-born neuroblasts during neurogenesis (Kanai et al., 2005). Overall, we propose that in the basal telencephalon COUP-TFI plays a critical temporal and spatial control over the differentiation of different subtypes of cortical interneurons, thereby enabling the temporal and spatial specification of PV-, and bipolar CR- and VIP-expressing interneurons.

\section{Increased inhibition mediated by altered balance of cortical interneuronal types might account for epilepsy resistance}

The role of GABAergic interneurons in the proper operation of cortical circuitry is widely recognized. By means of a precise somatodendritic arrangement of their synaptic contacts, diverse types of interneurons specifically control excitability of principal cells and other neuronal types (Markram et al., 2004; Ascoli et al., 2008), being responsible of setting brain rhythms and local field potential oscillations (Buzsáki and Draguhn, 2004). We know that GABAergic cell dysfunction is associated primarily with epilepsy, one of the most frequent neurological disorders occurring in the young population (Cossart et al., 2005; Baraban, 2007). However, we still lack a comprehensive understanding of what determines the expression of a particular phenotype and whether interneuronal dysfunction is cause or consequence of epileptic seizures.

Recent genetic models aimed to study the development of cortical interneurons have given us additional cues to draw this picture (Fig. 7). They constitute a unique tool because in most cases changes of subtype proportions precede pathological manifestations (Fig. 7B). Mice lacking the transcription factor Dlx1 suffer from an apoptotic loss of SST,$+ \mathrm{CR}+$, and NPY + subtypes, whereas PV+ interneurons were spared (Cobos et al., 2005). This reduction was associated with decreased GABAergic synaptic activity, distorted theta oscillations, and generalized spontaneous seizures, suggesting that less SST-, CR-, and NPYexpressing subtypes alone can account for seizures when the number of PV + cells remains constant. Interestingly, Sox6 mutants, which have a reduction of $\mathrm{PV}+$ and SST + interneurons and a constant number of the VIP + and CR + subtypes, exhibit pathological oscillations in the delta and beta bands and an early epileptic phenotype onset, that is not compensated by increased number of NPY + interneurons (Azim et al., 2009; Batista-Brito et al., 2009). This critical role of PV + and SST + subtypes is confirmed by early loss-of-function experiments of Nkx2.1 in which an excess of VIP + and CR + cells are generated at the expense of PV- and SST-expressing interneuronal populations (Butt et al., 2008). As a consequence, juvenile mice exhibit generalized seizures. A late (E12.5) loss of $\mathrm{Nkx} 2.1$ function leaves SST + cells unaffected and causes the same interneuronal shift exclusively at the upper layers. Remarkably, although their seizure susceptibility was not directly tested, these mice did not exhibit spontaneous seizures (Butt et al., 2008)

Very interestingly, mice lacking cyclinD2, which have reduced density of PV + interneurons but normal density of SST + and other CGE-derived interneurons, display decreased inhibitory synaptic activity and enhanced cortical excitability, although no spontaneous seizures were recorded (Glickstein et al., 2007b). In contrast, 
mice with mutation of the gene encoding $U P A R$ (urokinase plasminogen activator receptor) display a large reduction of $\mathrm{PV}+$ cells exclusively, and have spontaneous generalized seizures, being also more susceptible to PTZ-induced seizures (Powell et al., 2003).

All these mouse models argue for a differential role of diverse interneuronal types in controlling cortical excitability and seizures. Our study moves forward and demonstrates that, in the presence of a higher number of PV + and NPY + interneurons, COUP-TFI mutants show no discernible cortical EEG abnormalities, but on the contrary are more resistant to pharmacologically induced seizures. Such a resistance is dependent on GABAergic signaling because it is abolished by blockage of $\mathrm{GABA}_{\mathrm{A}}$ and $\mathrm{GABA}_{\mathrm{B}}$ receptors. Surprisingly, we found no changes in the gamma region of the spectrum despite the increased number of PV + interneurons. However, we must keep in mind that coarse wire EEG recordings are not well suited to look at the local organization of cortical gamma rhythms, and probably our recordings remained too superficial to detect changes caused by increased numbers of PV + cells at deep layers in mutants. Obviously, the cortical microcircuit is extremely complex, and other factors, such as the layer specificity of interneuronal loss and the total number of affected cells, can constrain the emergence of epileptic phenotypes and rhythm distortion. Future detailed studies of the local field potentials using multisite recordings would help to look at the finest spatial scale of the neocortex.

Another potential source of enhanced inhibition in our COUP-TFI CKO-Dlx5/6 mice is the reduction of the control exerted by fewer CR+ and VIP+ interneurons acting over other superficial layer interneurons. CR + and VIP + interneurons are known to preferentially target other interneurons (Staiger et al., 2004; Dávid et al., 2007). Thus, under conditions of reduced number of $\mathrm{CR}+$ and VIP+ interneurons, pyramidal cells would not be relieved from inhibition, being less excitable in COUP-TFI mutant mice. However, this is unlikely because (1) similar decrease of CR+ interneurons in the $D l x l$ mutants results in hyperexcitability and seizures, probably aided by concomitant reduction in the number of NPY + and SST + cells (Cobos et al., 2005); and (2) increased CR + and VIP + cells, in the presence of reduced number of PV + interneurons, failed to rescue the epileptic phenotype of Nkx2.1 mutants (Butt et al., 2008). Indeed, synaptic potentials elicited by $\mathrm{CR}+$ multipolar and bipolar interneurons on their few cortical pyramidal targets are weaker than potentials initiated by $\mathrm{PV}+$ interneurons, which dominate thalamocortical and intralaminar feedforward inhibition (Sun et al., 2006; Caputi et al., 2009; Xu and Callaway, 2009).
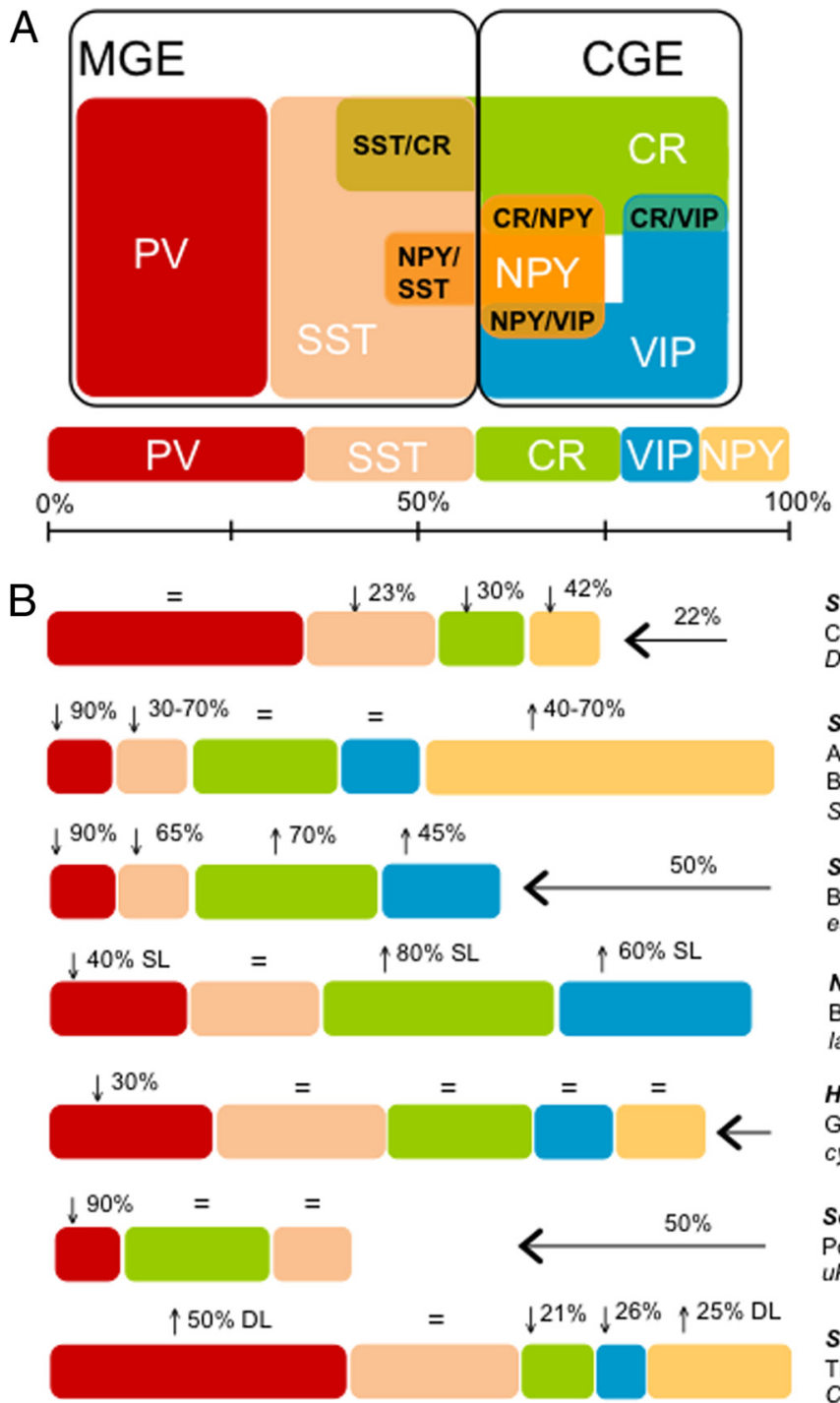

Seizures

Cobos et al. 2003 Dix1

Seizures

Azim et al. 2009

Batista-Brico et al. 2009

SOX 6

Seizures

Butt et al. 2008

early Nkx2.1 remova

No spontaneous seizures

Butt et al. 2008

late $N k \times 2.1$ removal

\section{Hyperexcitability}

Glickstein et al. 2007

cyclinD2

Seizures

Powell et al. 2003

UPAR

Seizure resistance

This study

COUP-TFI

Figure 7. Summary on the relationship between different interneuronal subpopulations and epileptic phenotypes. A, Schematic showing the proportion of the different cortical interneuronal subpopulations classified according to their molecular expres which together with SST-positive cells $(\sim 18 \%)$ mainly derive from the MGE. Instead, CR and VIP-expressing interneurons origimarkers colocalize, with SST/CR, SST/NPY, VIP/CR, and VIP/NPY being the most common. Data are from Gonchar and Burkhalter manipulations in the different papers cited at right and the corresponding clinical phenotype. $=$, Similar proportions compared with control; $\uparrow$, an increased proportion; $\downarrow$, decrease.

We also detected an increase of NPY + interneurons in the deep neocortical layers of COUP-TFI mutants. This probably reflects a population of NPY + interneurons, which derive from the dorsal MGE (Fogarty et al., 2007). NPY+ interneurons constitute a heterogeneous group, as this protein has been detected in a variety of cortical interneurons (Karagiannis et al., 2009), although a more homogeneous fraction appears to be generated from the preoptic area (Gelman et al., 2009). Interestingly, $\mathrm{NPY}+$ interneurons known to coexpress nitric oxide synthase (NOS) can be morphologically and physiologically identified as neurogliaform cells and share mainly an MGE origin, although a subpopulation of NPY + neurogliaform cells do not express NOS and derive from the CGE (Tricoire et al., 2010). Neurogliaform interneurons are a unique source of $\mathrm{GABA}_{\mathrm{B}}$-mediated inhibition 
for pyramidal cells (Tamás et al., 2003). If some of the exceeding $\mathrm{NPY}+$ interneurons detected in the lower cortical layers of COUP-TFI mutants correspond with neurogliaform cells, their possible role in controlling cortical excitability is intriguing. Indeed, we found that removal of $\mathrm{GABA}_{\mathrm{B}}$ inhibition in the presence of a $\mathrm{GABA}_{\mathrm{A}}$ antagonist was required to abolish differences of seizure onset between COUP-TFI mutants and WT mice. Although other sources of $\mathrm{GABA}_{\mathrm{B}}$-mediated inhibition operate at both presynaptic and postsynaptic levels, the specific role of this recently discovered interneuron population remains unknown. Future studies will be essential to overcome the origin, identity, and function of this miscellaneous interneuronal subtype.

\section{References}

Anderson SA, Eisenstat DD, Shi L, Rubenstein JL (1997) Interneuron migration from basal forebrain to neocortex: dependence on Dlx genes. Science 278:474-476.

Armentano M, Filosa A, Andolfi G, Studer M (2006) COUP-TFI is required for the formation of commissural projections in the forebrain by regulating axonal growth. Development 133:4151-4162.

Armentano M, Chou SJ, Tomassy GS, Leingärtner A, O'Leary DD, Studer M (2007) COUP-TFI regulates the balance of cortical patterning between frontal/motor and sensory areas. Nat Neurosci 10:1277-1286.

Ascoli GA, Alonso-Nanclares L, Anderson SA, Barrionuevo G, BenavidesPiccione R, Burkhalter A, Buzsáki G, Cauli B, Defelipe J, Fairén A, Feldmeyer D, Fishell G, Fregnac Y, Freund TF, Gardner D, Gardner EP, Goldberg JH, Helmstaedter M, Hestrin S, Karube F, et al. (2008) Petilla terminology: nomenclature of features of GABAergic interneurons of the cerebral cortex. Nat Rev Neurosci 9:557-568.

Azim E, Jabaudon D, Fame RM, Macklis JD (2009) SOX6 controls dorsal progenitor identity and interneuron diversity during neocortical development. Nat Neurosci 12:1238-1247.

Baraban SC (2007) Emerging epilepsy models: insights from mice, flies, worms and fish. Curr Opin Neurol 20:164-168.

Batista-Brito R, Fishell G (2009) The developmental integration of cortical interneurons into a functional network. Curr Top Dev Biol 87:81-118.

Batista-Brito R, Rossignol E, Hjerling-Leffler J, Denaxa M, Wegner M, Lefebvre V, Pachnis V, Fishell G (2009) The cell-intrinsic requirement of Sox6 for cortical interneuron development. Neuron 63:466-481.

Butt SJ, Fuccillo M, Nery S, Noctor S, Kriegstein A, Corbin JG, Fishell G (2005) The temporal and spatial origins of cortical interneurons predict their physiological subtype. Neuron 48:591-604.

Butt SJ, Sousa VH, Fuccillo MV, Hjerling-Leffler J, Miyoshi G, Kimura S, Fishell G (2008) The requirement of Nkx2-1 in the temporal specification of cortical interneuron subtypes. Neuron 59:722-732.

Buzsáki G, Draguhn A (2004) Neuronal oscillations in cortical networks. Science 304:1926-1929.

Caputi A, Rozov A, Blatow M, Monyer H (2009) Two calretinin-positive GABAergic cell types in layer $2 / 3$ of the mouse neocortex provide different forms of inhibition. Cereb Cortex 19:1345-1359.

Cobos I, Calcagnotto ME, Vilaythong AJ, Thwin MT, Noebels JL, Baraban SC, Rubenstein JL (2005) Mice lacking Dlx1 show subtype-specific loss of interneurons, reduced inhibition and epilepsy. Nat Neurosci 8:1059-1068.

Cobos I, Long JE, Thwin MT, Rubenstein JL (2006) Cellular patterns of transcription factor expression in developing cortical interneurons. Cereb Cortex 16 [Suppl 1]:i82-i88.

Corbin JG, Nery S, Fishell G (2001) Telencephalic cells take a tangent: nonradial migration in the mammalian forebrain. Nat Neurosci 4 [Suppl]:1177-1182.

Cossart R, Bernard C, Ben-Ari Y (2005) Multiple facets of GABAergic neurons and synapses: multiple fates of GABA signalling in epilepsies. Trends Neurosci 28:108-115.

Dávid C, Schleicher A, Zuschratter W, Staiger JF (2007) The innervation of parvalbumin-containing interneurons by VIP-immunopositive interneurons in the primary somatosensory cortex of the adult rat. Eur J Neurosci 25:2329-2340.

Du T, Xu Q, Ocbina PJ, Anderson SA (2008) NKX2.1 specifies cortical interneuron fate by activating Lhx6. Development 135:1559-1567.

Faedo A, Tomassy GS, Ruan Y, Teichmann H, Krauss S, Pleasure SJ, Tsai SY, Tsai MJ, Studer M, Rubenstein JL (2008) COUP-TFI coordinates corti- cal patterning, neurogenesis, and laminar fate and modulates MAPK/ ERK, AKT, and beta-catenin signaling. Cereb Cortex 18:2117-2131.

Flames N, Pla R, Gelman DM, Rubenstein JL, Puelles L, Marín O (2007) Delineation of multiple subpallial progenitor domains by the combinatorial expression of transcriptional codes. J Neurosci 27:9682-9695.

Fogarty M, Grist M, Gelman D, Marín O, Pachnis V, Kessaris N (2007) Spatial genetic patterning of the embryonic neuroepithelium generates GABAergic interneuron diversity in the adult cortex. J Neurosci 27:10935-10946.

Gelman DM, Martini FJ, Nóbrega-Pereira S, Pierani A, Kessaris N, Marín O (2009) The embryonic preoptic area is a novel source of cortical GABAergic interneurons. J Neurosci 29:9380-9389.

Glickstein SB, Alexander S, Ross ME (2007a) Differences in cyclin D2 and D1 protein expression distinguish forebrain progenitor subsets. Cereb Cortex 17:632-642.

Glickstein SB, Moore H, Slowinska B, Racchumi J, Suh M, Chuhma N, Ross ME (2007b) Selective cortical interneuron and GABA deficits in cyclin D2-null mice. Development 134:4083-4093.

Gonchar Y, Burkhalter A (1999) Connectivity of GABAergic calretininimmunoreactive neurons in rat primary visual cortex. Cereb Cortex 9:683-696.

Gröticke I, Hoffmann K, Löscher W (2007) Behavioral alterations in the pilocarpine model of temporal lobe epilepsy in mice. Exp Neurol 207:329-349.

Kanai MI, Okabe M, Hiromi Y (2005) seven-up controls switching of transcription factors that specify temporal identities of Drosophila neuroblasts. Dev Cell 8:203-213.

Kanatani S, Yozu M, Tabata H, Nakajima K (2008) COUP-TFII is preferentially expressed in the caudal ganglionic eminence and is involved in the caudal migratory stream. J Neurosci 28:13582-13591.

Karagiannis A, Gallopin T, Dávid C, Battaglia D, Geoffroy H, Rossier J, Hillman EM, Staiger JF, Cauli B (2009) Classification of NPY-expressing neocortical interneurons. J Neurosci 29:3642-3659.

Karlsson G, Kolb C, Hausdorf A, Portet C, Schmutz M, Olpe HR (1992) $\mathrm{GABA}_{\mathrm{B}}$ receptors in various in vitro and in vivo models of epilepsy: a study with the $\mathrm{GABA}_{\mathrm{B}}$ receptor blocker CGP 35348. Neuroscience $47: 63-68$

Kawaguchi Y, Kubota Y (1997) GABAergic cell subtypes and their synaptic connections in rat frontal cortex. Cereb Cortex 7:476-486.

Liodis P, Denaxa M, Grigoriou M, Akufo-Addo C, Yanagawa Y, Pachnis V (2007) Lhx6 activity is required for the normal migration and specification of cortical interneuron subtypes. J Neurosci 27:3078-3089.

Löscher W, Hönack D, Fassbender CP, Nolting B (1991) The role of technical, biological and pharmacological factors in the laboratory evaluation of anticonvulsant drugs. III. Pentylenetetrazole seizure models. Epilepsy Res 8:171-189.

Marín O, Rubenstein JL (2001) A long, remarkable journey: tangential migration in the telencephalon. Nat Rev Neurosci 2:780-790.

Marín O, Rubenstein JL (2003) Cell migration in the forebrain. Annu Rev Neurosci 26:441-483.

Markram H, Toledo-Rodriguez M, Wang Y, Gupta A, Silberberg G, Wu C (2004) Interneurons of the neocortical inhibitory system. Nat Rev Neurosci 5:793-807.

Miyoshi G, Butt SJ, Takebayashi H, Fishell G (2007) Physiologically distinct temporal cohorts of cortical interneurons arise from telencephalic Olig2expressing precursors. J Neurosci 27:7786-7798.

Miyoshi G, Hjerling-Leffler J, Karayannis T, Sousa VH, Butt SJ, Battiste J, Johnson JE, Machold RP, Fishell G (2010) Genetic fate mapping reveals that the caudal ganglionic eminence produces a large and diverse population of superficial cortical interneurons. J Neurosci 30:1582-1594.

Naka H, Nakamura S, Shimazaki T, Okano H (2008) Requirement for COUP-TFI and II in the temporal specification of neural stem cells in CNS development. Nat Neurosci 11:1014-1023.

Nery S, Fishell G, Corbin JG (2002) The caudal ganglionic eminence is a source of distinct cortical and subcortical cell populations. Nat Neurosci 5:1279-1287.

Nóbrega-Pereira S, Kessaris N, Du T, Kimura S, Anderson SA, Marín O (2008) Postmitotic Nkx2-1 controls the migration of telencephalic interneurons by direct repression of guidance receptors. Neuron 59:733-745.

Powell EM, Campbell DB, Stanwood GD, Davis C, Noebels JL, Levitt P (2003) Genetic disruption of cortical interneuron development causes 
region- and GABA cell type-specific deficits, epilepsy, and behavioral dysfunction. J Neurosci 23:622-631.

Sousa VH, Miyoshi G, Hjerling-Leffler J, Karayannis T, Fishell G (2009) Characterization of Nkx6-2-derived neocortical interneuron lineages. Cereb Cortex 19 [Suppl 1]:i1-i10.

Staiger JF, Masanneck C, Schleicher A, Zuschratter W (2004) Calbindincontaining interneurons are a target for VIP-immunoreactive synapses in rat primary somatosensory cortex. J Comp Neurol 468:179-189.

Stenman J, Toresson H, Campbell K (2003) Identification of two distinct progenitor populations in the lateral ganglionic eminence: implications for striatal and olfactory bulb neurogenesis. J Neurosci 23:167-174.

Sun QQ, Huguenard JR, Prince DA (2006) Barrel cortex microcircuits: thalamocortical feedforward inhibition in spiny stellate cells is mediated by a small number of fast-spiking interneurons. J Neurosci 26:1219-1230.

Sussel L, Marín O, Kimura S, Rubenstein JL (1999) Loss of Nkx2.1 homeobox gene function results in a ventral to dorsal molecular respecification within the basal telencephalon: evidence for a transformation of the pallidum into the striatum. Development 126:3359-3370.

Tamás G, Lorincz A, Simon A, Szabadics J (2003) Identified sources and targets of slow inhibition in the neocortex. Science 299:19021905.

Tomassy GS, De Leonibus E, Jabaudon D, Lodato S, Alfano C, Mele A, Mack- lis JD, Studer M (2010) Area-specific temporal control of corticospinal motor neuron differentiation by COUP-TFI. Proc Natl Acad Sci U S A 107:3576-3581.

Tricoire L, Pelkey KA, Daw MI, Sousa VH, Miyoshi G, Jeffries B, Cauli B, Fishell G, McBain CJ (2010) Common origins of hippocampal Ivy and nitric oxide synthase expressing neurogliaform cells. J Neurosci 30:2165-2176.

Tripodi M, Filosa A, Armentano M, Studer M (2004) The COUP-TF nuclear receptors regulate cell migration in the mammalian basal forebrain. Development 131:6119-6129.

Willi-Monnerat S, Migliavacca E, Surdez D, Delorenzi M, Luthi-Carter R, Terskikh AV (2008) Comprehensive spatiotemporal transcriptomic analyses of the ganglionic eminences demonstrate the uniqueness of its caudal subdivision. Mol Cell Neurosci 37:845-856.

Wonders CP, Anderson SA (2006) The origin and specification of cortical interneurons. Nat Rev Neurosci 7:687-696.

Xu Q, Cobos I, De La Cruz E, Rubenstein JL, Anderson SA (2004) Origins of cortical interneuron subtypes. J Neurosci 24:2612-2622.

Xu X, Callaway EM (2009) Laminar specificity of functional input to distinct types of inhibitory cortical neurons. J Neurosci 29:70-85.

Yozu M, Tabata H, Nakajima K (2005) The caudal migratory stream: a novel migratory stream of interneurons derived from the caudal ganglionic eminence in the developing mouse forebrain. J Neurosci 25:7268-7277. 